\title{
Functionally Altered Ruthenaindenes with Electron-rich and Electron-poor substituents
}

\author{
Hsiu L. Li, ${ }^{\dagger}$ Synøve Ø. Scottwell, James D. Watson, ${ }^{\dagger}$ Timothy E. Elton, ${ }^{\dagger}$ Hon C. Yu,
} Mohan Bhadbhade, ${ }^{\ddagger}$ Leslie D. Field,,$^{* *}$

† School of Chemistry, ${ }^{\ddagger}$ Mark Wainwright Analytical Centre, University of New South Wales, NSW 2052, Australia;

\section{Supporting Information}

\section{CONTENTS}

\begin{tabular}{|c|c|c|}
\hline & & Page \\
\hline Com & nd numbering & $\mathrm{S}_{3}$ \\
\hline NMF & d high resolution mass spectra & $\mathrm{S}_{4}$ \\
\hline S2.1 & {$\left[\mathrm{Ru}\left(\eta^{3}-\mathrm{MeO}-4-\mathrm{C}_{6} \mathrm{H}_{4} \mathrm{C} \equiv \mathrm{C}-\mathrm{C}=\mathrm{CH}\left(\mathrm{C}_{6} \mathrm{H}_{4}-4-\mathrm{OMe}\right)\right)\left(\mathrm{PMe}_{3}\right)_{4}\right]^{+} \mathrm{BPh}_{4}^{-} \mathbf{1 a}-\mathbf{B P h}_{4}$} & $\mathrm{~S}_{4}$ \\
\hline & Figure S1. ${ }^{31} \mathrm{P}\left\{{ }^{1} \mathrm{H}\right\}$ NMR spectrum & $\mathrm{S}_{4}$ \\
\hline & Figure S2. ${ }^{1} \mathrm{H}$ NMR spectrum & $\mathrm{S}_{4}$ \\
\hline S2.2 & {$\left[\mathrm{Ru}\left(\eta^{3}-\mathrm{MeO}-4-\mathrm{C}_{6} \mathrm{H}_{4} \mathrm{C} \equiv \mathrm{C}-\mathrm{C}=\mathrm{CH}\left(\mathrm{C}_{6} \mathrm{H}_{4}-4-\mathrm{OMe}\right)\right)\left(\mathrm{PMe}_{3}\right)_{4}\right]^{+} \mathrm{BF}_{4}^{-} \mathbf{1 a}-\mathbf{B F}_{4}$} & $\mathrm{~S}_{5}$ \\
\hline & Figure $\mathrm{S}_{3} .{ }^{3} \mathrm{P}\left\{{ }^{1} \mathrm{H}\right\}$ NMR spectrum & $\mathrm{S}_{5}$ \\
\hline & Figure $\mathrm{S}_{4} .{ }^{1} \mathrm{H}$ NMR spectrum & $\mathrm{S}_{5}$ \\
\hline & Figure $\mathrm{S}_{5} .{ }^{13} \mathrm{C}\left\{{ }^{1} \mathrm{H}\right\}$ NMR spectrum & S6 \\
\hline & Figure S6. ${ }^{19} \mathrm{~F}$ NMR spectrum & S6 \\
\hline & Figure $\mathrm{S}_{7}$. HRMS & $\mathrm{S}_{7}$ \\
\hline & Figure S8. HRMS (expansion) & S8 \\
\hline S2.3 & {$\left[\mathrm{Ru}\left(\eta^{3}-\mathrm{PhC} \equiv \mathrm{C}-\mathrm{C}=\mathrm{CH}(\mathrm{Ph})\right)\left(\mathrm{PMe}_{3}\right)_{4}\right]^{+} \mathrm{BPh}_{4}^{-} \mathbf{1 b}$} & S9 \\
\hline & Figure $\mathrm{S}_{9 .}{ }^{31} \mathrm{P}\left\{{ }^{1} \mathrm{H}\right\}$ NMR spectrum & S9 \\
\hline & Figure Sio. ${ }^{1} \mathrm{H}$ NMR spectrum & S9 \\
\hline S2.4 & {$\left[\mathrm{Ru}\left(\eta^{3}-\mathrm{CF}_{3}-4-\mathrm{C}_{6} \mathrm{H}_{4} \mathrm{C} \equiv \mathrm{C}-\mathrm{C}=\mathrm{CH}\left(\mathrm{C}_{6} \mathrm{H}_{4}-4-\mathrm{CF}_{3}\right)\right)\left(\mathrm{PMe}_{3}\right)_{4}\right]^{+} \mathrm{BPh}_{4}^{-} \mathbf{1 c}-\mathbf{B P h}_{4}$} & S10 \\
\hline & Figure S11. ${ }^{31} \mathrm{P}\left\{{ }^{1} \mathrm{H}\right\}$ NMR spectrum & S10 \\
\hline & Figure S12. ${ }^{1} \mathrm{H}$ NMR spectrum & S10 \\
\hline & Figure S13. ${ }^{19} \mathrm{~F}$ NMR spectrum & S11 \\
\hline S2.5 & {$\left[\mathrm{Ru}\left(\eta^{3}-\mathrm{CF}_{3}-4-\mathrm{C}_{6} \mathrm{H}_{4} \mathrm{C} \equiv \mathrm{C}-\mathrm{C}=\mathrm{CH}\left(\mathrm{C}_{6} \mathrm{H}_{4}-4-\mathrm{CF}_{3}\right)\right)\left(\mathrm{PMe}_{3}\right)_{4}\right]^{+} \mathrm{BF}_{4}^{-} \mathbf{1 c}-\mathbf{B F}_{4}$} & S11 \\
\hline & Figure $\mathrm{S}_{14} .{ }^{31} \mathrm{P}\left\{{ }^{1} \mathrm{H}\right\}$ NMR spectrum & S11 \\
\hline & Figure $\mathrm{S}_{15} .{ }^{1} \mathrm{H}$ NMR spectrum & $\mathrm{S} 12$ \\
\hline & Figure $\mathrm{S} 16 .{ }^{13} \mathrm{C}\left\{{ }^{1} \mathrm{H}\right\}$ NMR spectrum & $\mathrm{S} 12$ \\
\hline & Figure S17. ${ }^{19} \mathrm{~F}$ NMR spectrum & $\mathrm{S} 13$ \\
\hline S2.6 & {$\left[\mathrm{Ru}\left(\mathrm{MeO}-4-\mathrm{C}_{6} \mathrm{H}_{4} \mathrm{C} \equiv \mathrm{C}-\mathrm{C}=\mathrm{CH}\left(\mathrm{C}_{6} \mathrm{H}_{3}-4-\mathrm{OMe}\right)\left(\mathrm{PMe}_{3}\right)_{4}\right] \mathbf{2 a}\right.$} & $\mathrm{S}_{13}$ \\
\hline & Figure $\mathrm{S} 18 .{ }^{31} \mathrm{P}\left\{{ }^{1} \mathrm{H}\right\}$ NMR spectrum & $\mathrm{S} 13$ \\
\hline & Figure S19. ${ }^{1} \mathrm{H}$ NMR spectrum & $\mathrm{S} 14$ \\
\hline & Figure S2o. ${ }^{13} \mathrm{C}\left\{{ }^{1} \mathrm{H}\right\}$ NMR spectrum & $\mathrm{S}_{14}$ \\
\hline & Figure S21. HRMS & $\mathrm{S}_{15}$ \\
\hline & Figure S22. HRMS (expansion) & S16 \\
\hline S2.7 & {$\left[\mathrm{Ru}\left(\mathrm{CF}_{3}-4-\mathrm{C}_{6} \mathrm{H}_{4} \mathrm{C} \equiv \mathrm{C}-\mathrm{C}=\mathrm{CH}\left(\mathrm{C}_{6} \mathrm{H}_{3}-4-\mathrm{CF}_{3}\right)\left(\mathrm{PMe}_{3}\right)_{4}\right] \mathbf{2 c}\right.$} & $\mathrm{S} 17$ \\
\hline & Figure $\mathrm{S}_{23} \cdot{ }^{31} \mathrm{P}\left\{{ }^{1} \mathrm{H}\right\}$ NMR spectrum & $\mathrm{S} 17$ \\
\hline & Figure S24. ${ }^{1} \mathrm{H}$ NMR spectrum & $\mathrm{S} 17$ \\
\hline & Figure $\mathrm{S}_{25} .{ }^{13} \mathrm{C}\left\{{ }^{1} \mathrm{H}\right\}$ NMR spectrum & S18 \\
\hline & Figure S26. ${ }^{19} \mathrm{~F}$ NMR spectrum & $\mathrm{S} 18$ \\
\hline & Figure S27. HRMS & S19 \\
\hline & Figure S28. HRMS (expansion) & S2O \\
\hline S2.8 & cis/trans- $\left[\mathrm{Ru}\left(\mathrm{C} \equiv \mathrm{CC}_{6} \mathrm{H}_{4}-4-\mathrm{OMe}\right)_{2}\left(\mathrm{PMe}_{3}\right)_{4}\right] \mathbf{3 a}^{\mathbf{a}}$ & $S_{21}$ \\
\hline
\end{tabular}




\begin{tabular}{|c|c|c|}
\hline \multicolumn{2}{|r|}{ Figure S29. ${ }^{31} \mathrm{P}\left\{{ }^{1} \mathrm{H}\right\}$ NMR spectrum } & $\mathrm{S}_{21}$ \\
\hline \multicolumn{2}{|r|}{ Figure $\mathrm{S}_{30} .{ }^{1} \mathrm{H}$ NMR spectrum } & $\mathrm{S}_{21}$ \\
\hline \multicolumn{2}{|r|}{ Figure $\mathrm{S}_{31 .}{ }^{13} \mathrm{C}\left\{{ }^{1} \mathrm{H}\right\}$ NMR spectrum } & S22 \\
\hline \multicolumn{2}{|r|}{ Figure S32. HRMS } & S22 \\
\hline \multicolumn{2}{|r|}{ Figure 333. HRMS (expansion) } & $\mathrm{S}_{23}$ \\
\hline \multirow[t]{5}{*}{ S2.9 } & cis/trans- $\left.\left[\mathrm{Ru}\left(\mathrm{C} \equiv \mathrm{CC}_{6} \mathrm{H}_{4}-4-\mathrm{CF}_{3}\right)_{2}\left(\mathrm{PMe}_{3}\right)_{4}\right]\right]_{3} \mathrm{C}$ & $\mathrm{S}_{24}$ \\
\hline & Figure $\mathrm{S}_{34} \cdot{ }^{31} \mathrm{P}\left\{{ }^{1} \mathrm{H}\right\}$ NMR spectrum & $\mathrm{S} 24$ \\
\hline & Figure $\mathrm{S}_{35} .{ }^{1} \mathrm{H}$ NMR spectrum & $\mathrm{S} 24$ \\
\hline & Figure $\mathrm{S}_{3} 6 .{ }^{13} \mathrm{C}\left\{{ }^{1} \mathrm{H}\right\}$ NMR spectrum & $\mathrm{S} 25$ \\
\hline & Figure $\mathrm{S}_{37}{ }^{19} \mathrm{~F}$ NMR spectrum & $\mathrm{S}_{25}$ \\
\hline \multirow[t]{7}{*}{ S2.10 } & trans $-\left[\mathrm{Ru}\left(=\mathrm{C}=\mathrm{CH}\left(\mathrm{C}_{6} \mathrm{H}_{4}-4-\mathrm{OMe}\right)\right)\left(\mathrm{C}=\mathrm{CC}_{6} \mathrm{H}_{4}-4-\mathrm{OMe}\right)\left(\mathrm{PMe}_{3}\right)_{4}\right]^{+} \mathrm{BF}_{4}^{-} \mathbf{4 a}^{-}$ & S26 \\
\hline & Figure $\mathrm{S}_{3} 8 .{ }^{1} \mathrm{P}\left\{{ }^{1} \mathrm{H}\right\}$ NMR spectrum & $\mathrm{S}_{26}$ \\
\hline & Figure $\mathrm{S}_{39 .}{ }^{1} \mathrm{H}$ NMR spectrum & S26 \\
\hline & Figure $\mathrm{S}_{40} .{ }^{13} \mathrm{C}\left\{{ }^{1} \mathrm{H}\right\}$ NMR spectrum & S27 \\
\hline & Figure $\mathrm{S}_{41 .}{ }^{19} \mathrm{~F}$ NMR spectrum & S27 \\
\hline & Figure $\mathrm{S}_{42} . \mathrm{HRMS}$ & $\mathrm{S} 28$ \\
\hline & Figure S43. HRMS (expansion) & S29 \\
\hline \multirow[t]{5}{*}{ S2.11 } & trans $-\left[\mathrm{Ru}(=\mathrm{C}=\mathrm{CH}(\mathrm{Ph}))(\mathrm{C} \equiv \mathrm{CPh})\left(\mathrm{PMe}_{3}\right)_{4}\right]^{+} \mathrm{BF}_{4}^{-} \mathbf{4} \mathbf{b}$ & $\mathrm{S}_{30}$ \\
\hline & Figure $\mathrm{S}_{44} \cdot{ }^{31} \mathrm{P}\left\{{ }^{1} \mathrm{H}\right\}$ NMR spectrum & S30 \\
\hline & Figure $\mathrm{S}_{45} \cdot{ }^{1} \mathrm{H}$ NMR spectrum & $\mathrm{S}_{30}$ \\
\hline & Figure $\mathrm{S}_{4} 6 .{ }^{13} \mathrm{C}\left\{{ }^{1} \mathrm{H}\right\}$ NMR spectrum & $\mathrm{S}_{31}$ \\
\hline & Figure S47. ${ }^{19} \mathrm{~F}$ NMR spectrum & $\mathrm{S}_{31}$ \\
\hline \multirow[t]{2}{*}{ X-ray } & stal structure of trans- $\left[\mathrm{Ru}\left(=\mathrm{C}=\mathrm{CH}\left(\mathrm{C}_{6} \mathrm{H}_{4}-4-\mathrm{OMe}\right)\right)\left(\mathrm{C} \equiv \mathrm{CC}_{6} \mathrm{H}_{4}-4-\mathrm{OMe}\right)\left(\mathrm{PMe}_{3}\right)_{4}\right]^{+} \mathrm{BF}_{4}^{-} \mathbf{4}^{\mathbf{a}}$ & $\mathrm{S}_{32}$ \\
\hline & Figure $\mathrm{S}_{48}$. ORTEP & $\mathrm{S}_{32}$ \\
\hline \multicolumn{2}{|c|}{ Table S1 Crystallographic data for $\mathbf{1 c}, \mathbf{2 a}, \mathbf{3} \mathbf{c}, \mathbf{4} \mathbf{a}$ and $\mathbf{4} \mathbf{b}$} & $\mathrm{S}_{33}$ \\
\hline
\end{tabular}




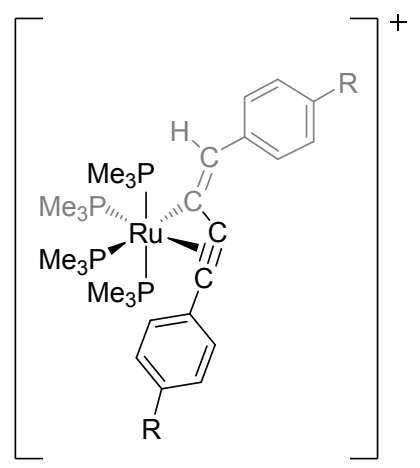

1a: $\mathrm{R}=\mathrm{OMe}$

1b: $\mathrm{R}=\mathrm{H}$

1c: $\mathrm{R}=\mathrm{CF}_{3}$

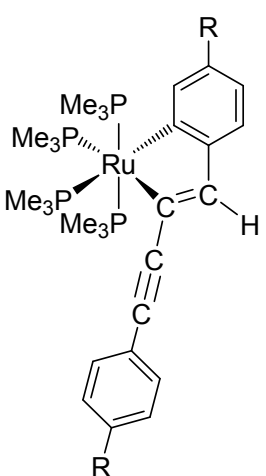

2a: $\mathrm{R}=\mathrm{OMe}$

2b: $\mathrm{R}=\mathrm{H}$

2c: $\mathrm{R}=\mathrm{CF}_{3}$
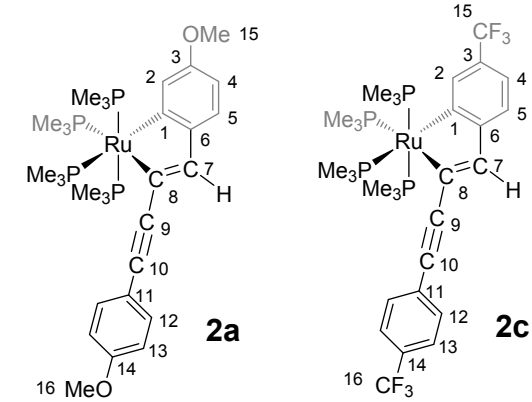

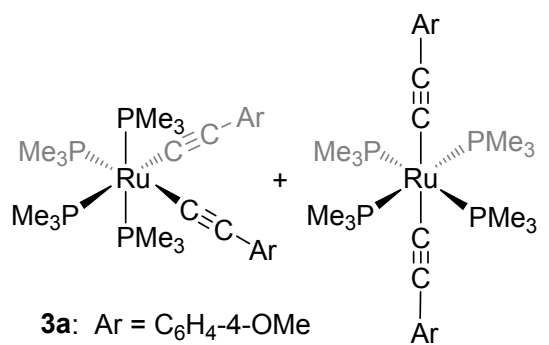

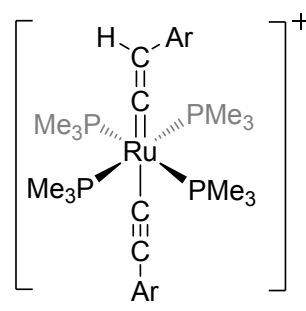

4a: $\mathrm{Ar}=\mathrm{C}_{6} \mathrm{H}_{4}-4-\mathrm{OMe}$

4b: $\mathrm{Ar}=\mathrm{Ph}$ 
S2. NMR AND HIGH RESOLUTION MASS SPECTRA

S2.1 $\left[\mathrm{Ru}\left(\eta^{3}-\mathrm{MeO}-4-\mathrm{C}_{6} \mathrm{H}_{4} \mathrm{C} \equiv \mathrm{C}-\mathrm{C}=\mathrm{CH}\left(\mathrm{C}_{6} \mathrm{H}_{4}-4-\mathrm{OMe}\right)\right)\left(\mathrm{PMe}_{3}\right)_{4}\right]^{+} \mathrm{BPh}_{4}{ }^{-}{ }^{1 a-}-\mathrm{BPh}_{4}$.

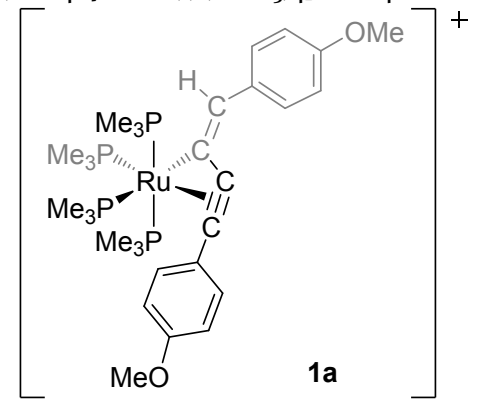

Figure S1. ${ }^{31} \mathrm{P}\left\{{ }^{1} \mathrm{H}\right\}$ NMR spectrum for $\left[\mathrm{Ru}\left(\eta^{3}-\mathrm{MeO}-4-\mathrm{C}_{6} \mathrm{H}_{4} \mathrm{C} \equiv \mathrm{C}-\mathrm{C}=\mathrm{CH}\left(\mathrm{C}_{6} \mathrm{H}_{4}-4-\mathrm{OMe}\right)\right)\left(\mathrm{PMe}_{3}\right)_{4}\right]+\mathrm{BPh}_{4}^{-} \quad \mathbf{1 a -}-\mathbf{B P h}_{4}$ (acetone- $d_{6}, 162 \mathrm{MHz}$ )

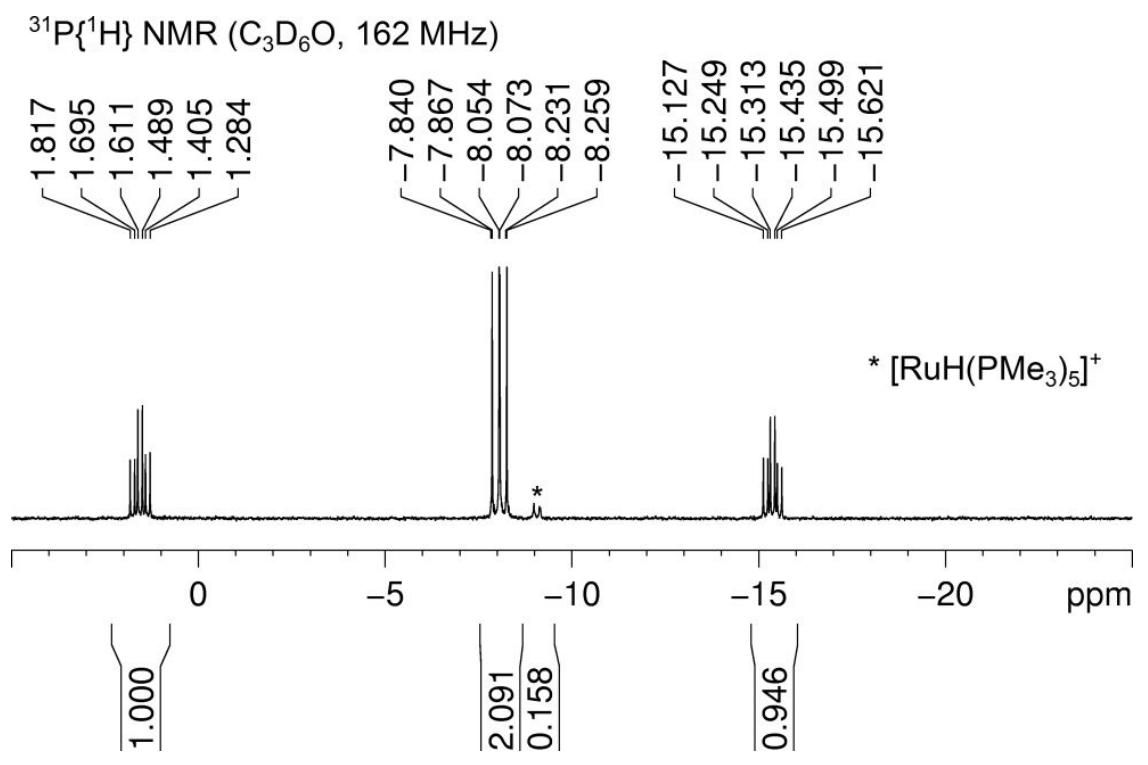

Figure S2. ${ }^{1} \mathrm{H} \quad \mathrm{NMR}$ spectrum for $\left[\mathrm{Ru}\left(\eta^{3}-\mathrm{MeO}-4-\mathrm{C}_{6} \mathrm{H}_{4} \mathrm{C} \equiv \mathrm{C}-\mathrm{C}=\mathrm{CH}\left(\mathrm{C}_{6} \mathrm{H}_{4}-4-\mathrm{OMe}\right)\right)\left(\mathrm{PMe}_{3}\right)_{4}\right]^{+} \mathrm{BPh}_{4}^{-} \quad \mathbf{1 a -}-\mathbf{B P h}_{4}$ (acetone- $d_{6}, 400 \mathrm{MHz}$ )

${ }^{1} \mathrm{H} \operatorname{NMR}\left(\mathrm{C}_{3} \mathrm{D}_{6} \mathrm{O}, 400 \mathrm{MHz}\right)$

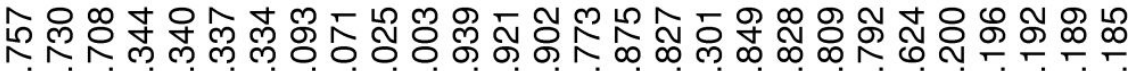

$\hat{N} N$ N N N

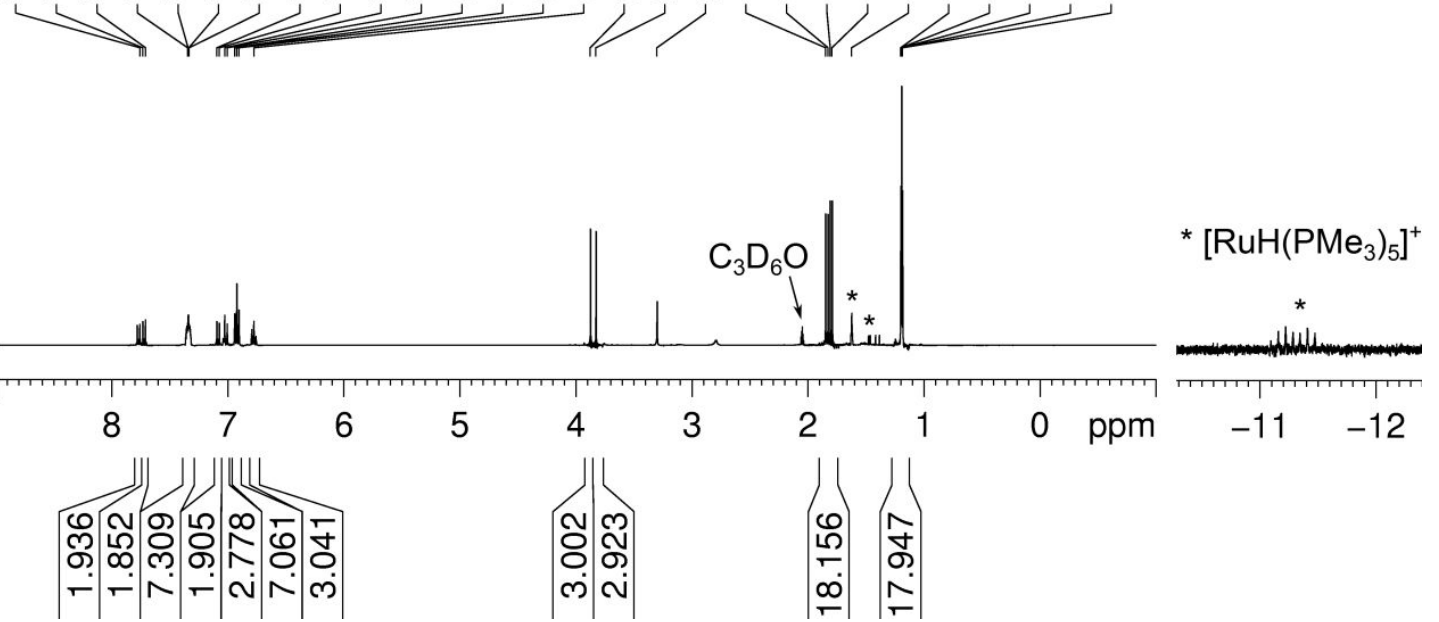


S2.2. $\quad\left[\mathrm{Ru}\left(\eta^{3}-\mathrm{MeO}-4-\mathrm{C}_{6} \mathrm{H}_{4} \mathrm{C} \equiv \mathrm{C}-\mathrm{C}=\mathrm{CH}\left(\mathrm{C}_{6} \mathrm{H}_{4}-4-\mathrm{OMe}\right)\right)\left(\mathrm{PMe}_{3}\right)_{4}\right]^{+} \mathrm{BF}_{4}^{-} \mathbf{1 a}^{-}-\mathrm{BF}_{4}$.

Figure S3. ${ }^{31} \mathrm{P}\left\{{ }_{1}^{1} \mathrm{H}\right\} \quad \mathrm{NMR}$ spectrum for $\left[\mathrm{Ru}\left(\eta \eta^{3}-\mathrm{MeO}-4-\mathrm{C}_{6} \mathrm{H}_{4} \mathrm{C} \equiv \mathrm{C}-\mathrm{C}=\mathrm{CH}\left(\mathrm{C}_{6} \mathrm{H}_{4}-4-\mathrm{OMe}\right)\right)\left(\mathrm{PMe}_{3}\right)_{4}\right]^{+} \mathrm{BF}_{4}^{-} \quad \mathbf{1 a}^{-}-\mathrm{BF}_{4}$ (acetone- $d_{6}, 243 \mathrm{MHz}$ )

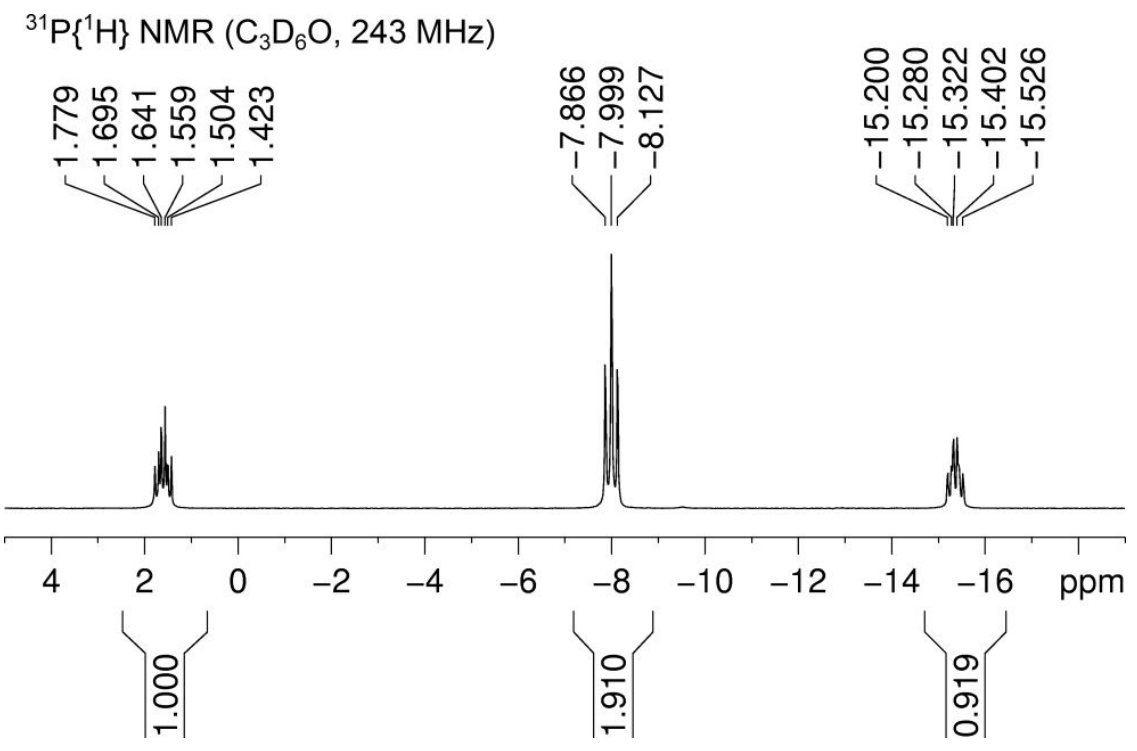

Figure $\mathrm{S}_{4} \cdot{ }^{1} \mathrm{H}$ NMR spectrum for $\left[\mathrm{Ru}\left(\eta \eta^{3}-\mathrm{MeO}-4-\mathrm{C}_{6} \mathrm{H}_{4} \mathrm{C} \equiv \mathrm{C}-\mathrm{C}=\mathrm{CH}\left(\mathrm{C}_{6} \mathrm{H}_{4}-4-\mathrm{OMe}\right)\right)\left(\mathrm{PMe}_{3}\right)_{4}\right]+\mathrm{BF}_{4}^{-}$1a- $\mathbf{B F}_{4}$ (acetone- $d_{6}$, $600 \mathrm{MHz}$ )

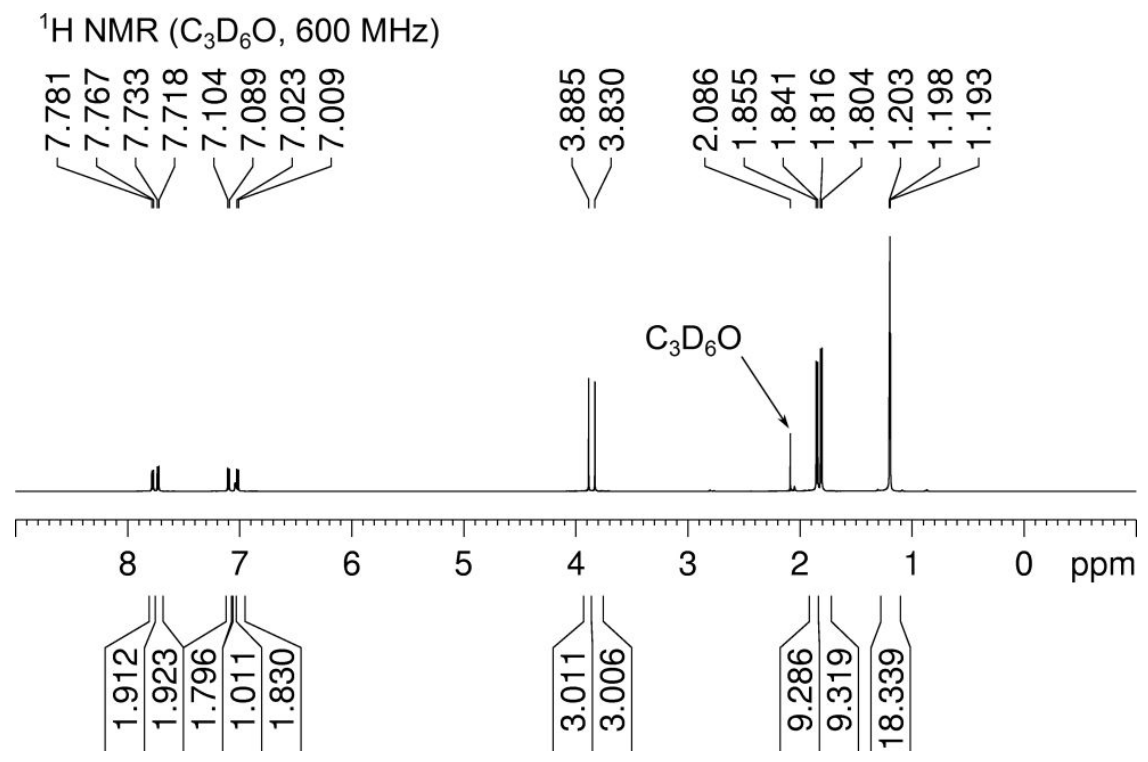


Figure $\mathrm{S}_{5} .{ }^{13} \mathrm{C}\left\{{ }^{1} \mathrm{H}\right\} \quad \mathrm{NMR}$ spectrum for $\left[\mathrm{Ru}\left(\eta^{3}-\mathrm{MeO}-4-\mathrm{C}_{6} \mathrm{H}_{4} \mathrm{C} \equiv \mathrm{C}-\mathrm{C}=\mathrm{CH}\left(\mathrm{C}_{6} \mathrm{H}_{4}-4-\mathrm{OMe}\right)\right)\left(\mathrm{PMe}_{3}\right)_{4}\right]^{+} \mathrm{BF}_{4}^{-} \quad \mathbf{1 a}^{-} \mathbf{B F}_{4}$ (acetone- $\left.d_{6}, 151 \mathrm{MHz}\right)$

${ }^{13} \mathrm{C}\left\{{ }^{1} \mathrm{H}\right\} \operatorname{NMR}\left(\mathrm{C}_{3} \mathrm{D}_{6} \mathrm{O}, 151 \mathrm{MHz}\right)$

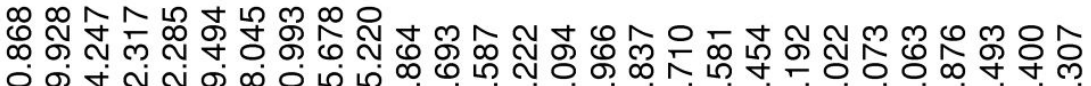

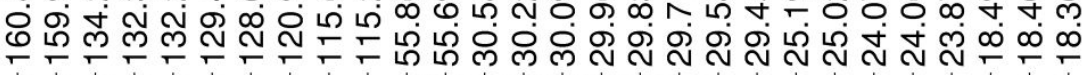

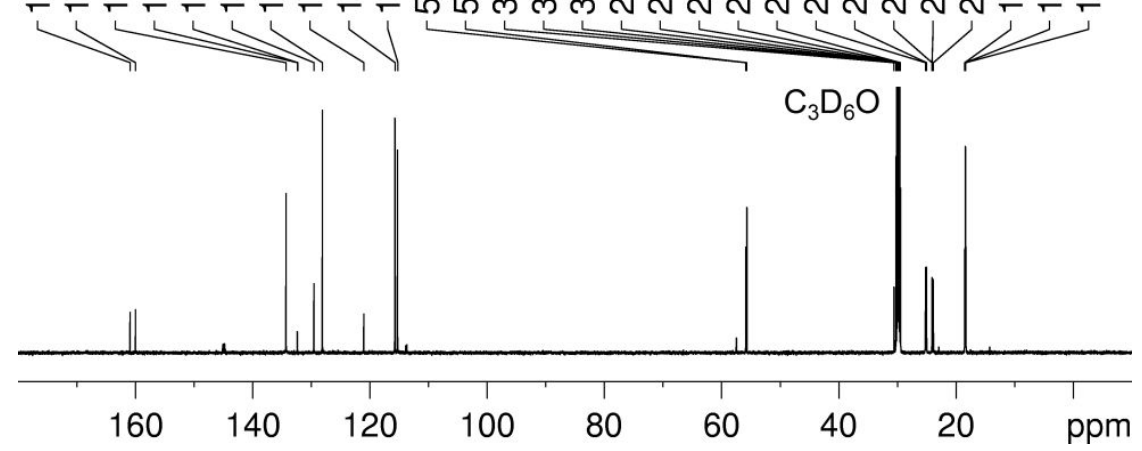

Figure S6. ${ }^{19} \mathrm{~F}$ NMR spectrum for $\left[\mathrm{Ru}\left(\eta^{3}-\mathrm{MeO}-4-\mathrm{C}_{6} \mathrm{H}_{4} \mathrm{C} \equiv \mathrm{C}-\mathrm{C}=\mathrm{CH}\left(\mathrm{C}_{6} \mathrm{H}_{4}-4-\mathrm{OMe}\right)\right)\left(\mathrm{PMe}_{3}\right)_{4}\right]^{+} \mathrm{BF}_{4}^{-}$1a- $\mathbf{B F}_{4}$ (acetone- $d_{6}$, $565 \mathrm{MHz}$ )

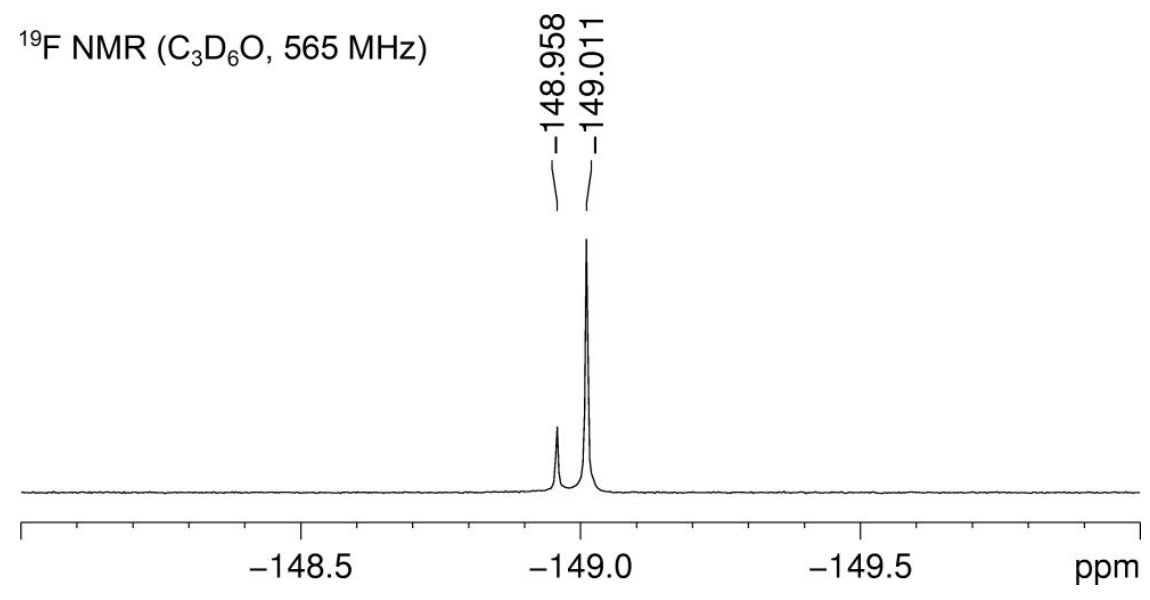


Figure $\mathrm{S}_{7}$. HRMS for $\left[\mathrm{Ru}\left(\eta^{3}-\mathrm{MeO}-4-\mathrm{C}_{6} \mathrm{H}_{4} \mathrm{C} \equiv \mathrm{C}-\mathrm{C}=\mathrm{CH}\left(\mathrm{C}_{6} \mathrm{H}_{4}-4-\mathrm{OMe}\right)\right)\left(\mathrm{PMe}_{3}\right)_{4}\right]^{+} \mathrm{BF}_{4}^{-}$la- $\mathbf{B F}_{4}$ (acetonitrile, ESI) 43-97-LHL_Pos_Full \#1-18 RT: 0.01-0.95 AV: 18 NL: 3.90E7 T: FTMS + p NSI Full ms [100.00-2000.00]

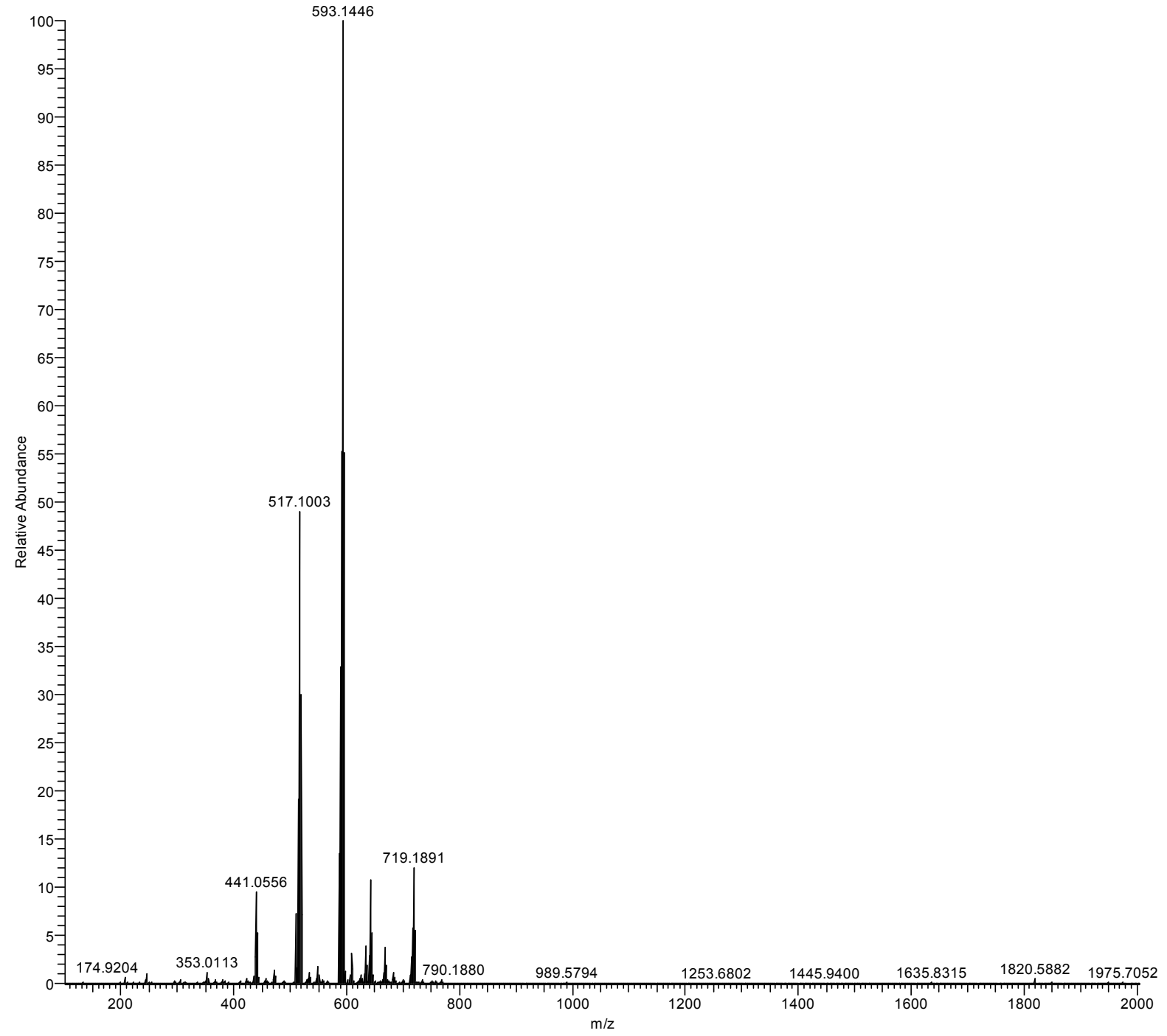


Figure S8. HRMS for $\left[\mathrm{Ru}\left(\eta^{3}-\mathrm{MeO}-4-\mathrm{C}_{6} \mathrm{H}_{4} \mathrm{C} \equiv \mathrm{C}-\mathrm{C}=\mathrm{CH}\left(\mathrm{C}_{6} \mathrm{H}_{4}-4-\mathrm{OMe}\right)\right)\left(\mathrm{PMe}_{3}\right)_{4}\right]^{+} \mathrm{BF}_{4}^{-} \mathbf{1 a}^{-} \mathbf{B F}_{4}$ (expansion, acetonitrile, ESI)

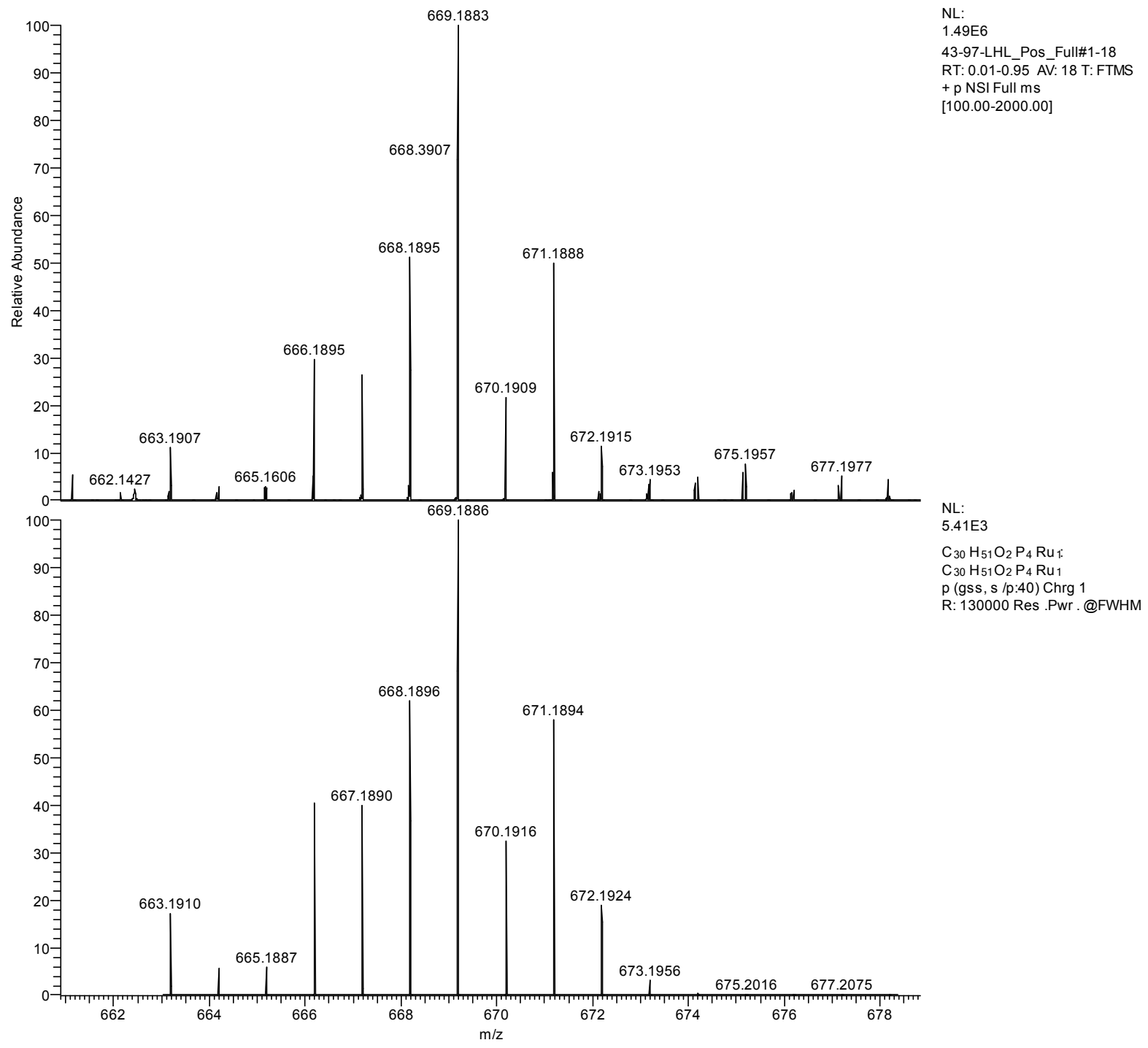




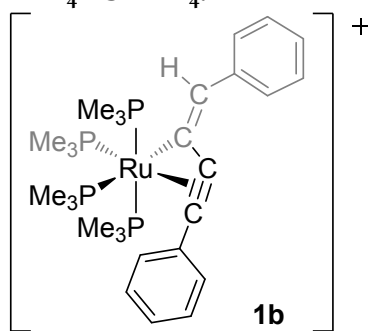

Figure S9. ${ }^{3} \mathrm{P}\left\{{ }_{1} \mathrm{H}\right\}$ NMR spectrum for $\left[\mathrm{Ru}\left(\eta{ }^{3}-\mathrm{PhC} \equiv \mathrm{C}-\mathrm{C}=\mathrm{CH}(\mathrm{Ph})\right)\left(\mathrm{PMe}_{3}\right)_{4}\right]+\mathrm{BPh}_{4}{ }^{-} \mathbf{1 b}-\mathbf{B P h}_{\mathbf{4}}$ (acetone- $\left.d_{6}, 243 \mathrm{MHz}\right)$ ${ }^{31} \mathrm{P}\left\{{ }^{1} \mathrm{H}\right\} \operatorname{NMR}\left(\mathrm{C}_{3} \mathrm{D}_{6} \mathrm{O}, 243 \mathrm{MHz}\right)$

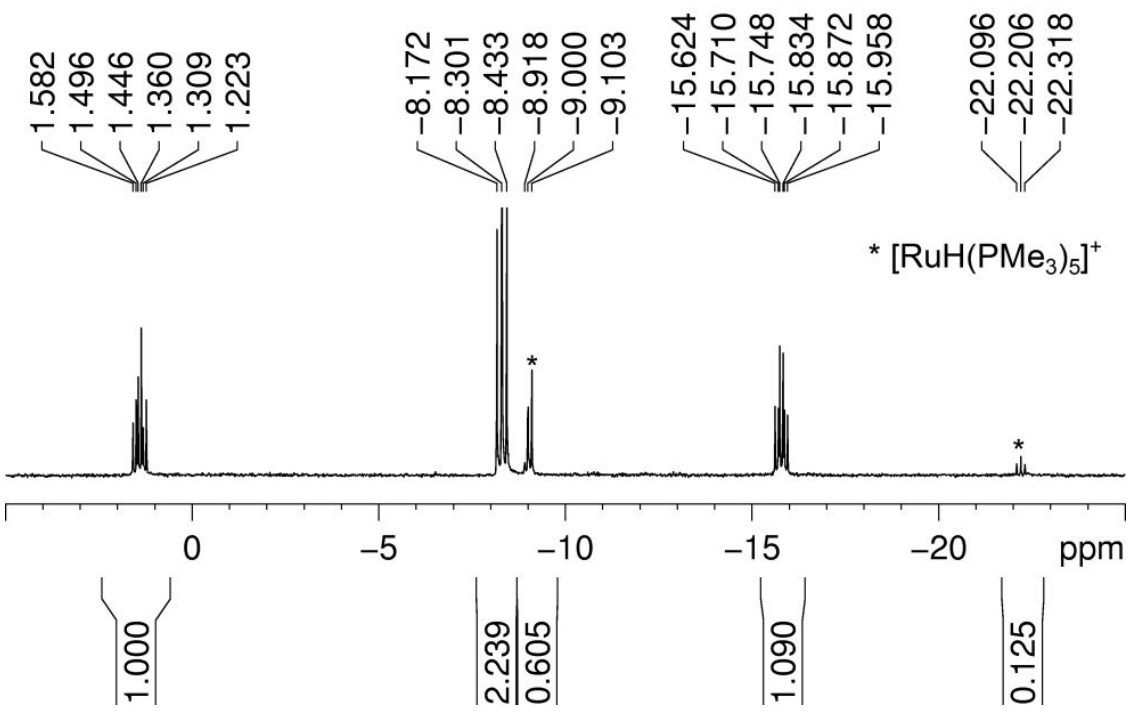

Figure S1o. ${ }^{1} \mathrm{H}$ NMR spectrum for $\left[\mathrm{Ru}\left(\eta^{3}-\mathrm{PhC} \equiv \mathrm{C}-\mathrm{C}=\mathrm{CH}(\mathrm{Ph})\right)\left(\mathrm{PMe}_{3}\right)_{4}\right]+\mathrm{BPh}_{4}{ }^{-} \mathbf{1 b}-\mathbf{B P h}_{4}$ (acetone- $d_{6}, 6$ oo $\left.\mathrm{MHz}\right)$ ${ }^{1} \mathrm{H} \operatorname{NMR}\left(\mathrm{C}_{3} \mathrm{D}_{6} \mathrm{O}, 600 \mathrm{MHz}\right)$

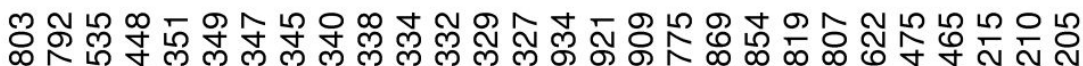

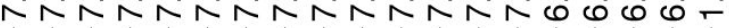

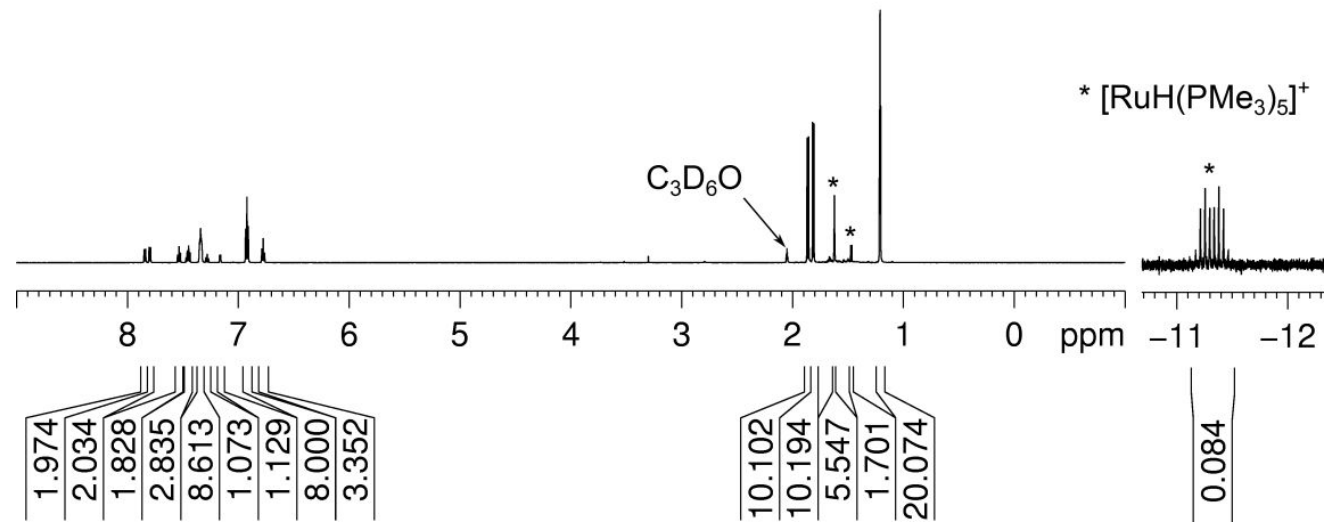


S2.4 $\left[\mathrm{Ru}\left(\eta^{3}-\mathrm{CF}_{3}-4-\mathrm{C}_{6} \mathrm{H}_{4} \mathrm{C} \equiv \mathrm{C}-\mathrm{C}=\mathrm{CH}\left(\mathrm{C}_{6} \mathrm{H}_{4}-4-\mathrm{CF}_{3}\right)\right)\left(\mathrm{PMe}_{3}\right)_{4}\right]^{+} \mathrm{BPh}_{4}^{-} \mathrm{IC}^{-\mathrm{BPh}_{4}}$.

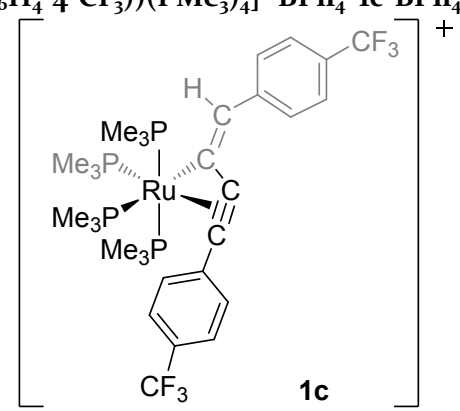

Figure S11. ${ }^{31} \mathrm{P}\left\{{ }^{1} \mathrm{H}\right\} \quad \mathrm{NMR}$ spectrum for $\left[\mathrm{Ru}\left(\eta^{3}-\mathrm{CF}_{3}-4-\mathrm{C}_{6} \mathrm{H}_{4} \mathrm{C} \equiv \mathrm{C}-\mathrm{C}=\mathrm{CH}\left(\mathrm{C}_{6} \mathrm{H}_{4}-4-\mathrm{CF}_{3}\right)\right)\left(\mathrm{PMe}_{3}\right)_{4}\right]^{+} \mathrm{BPh}_{4}^{-} \quad \mathbf{1 c}_{-}-\mathbf{B P h}_{4}$ (acetone- $d_{6}, 162 \mathrm{MHz}$ )

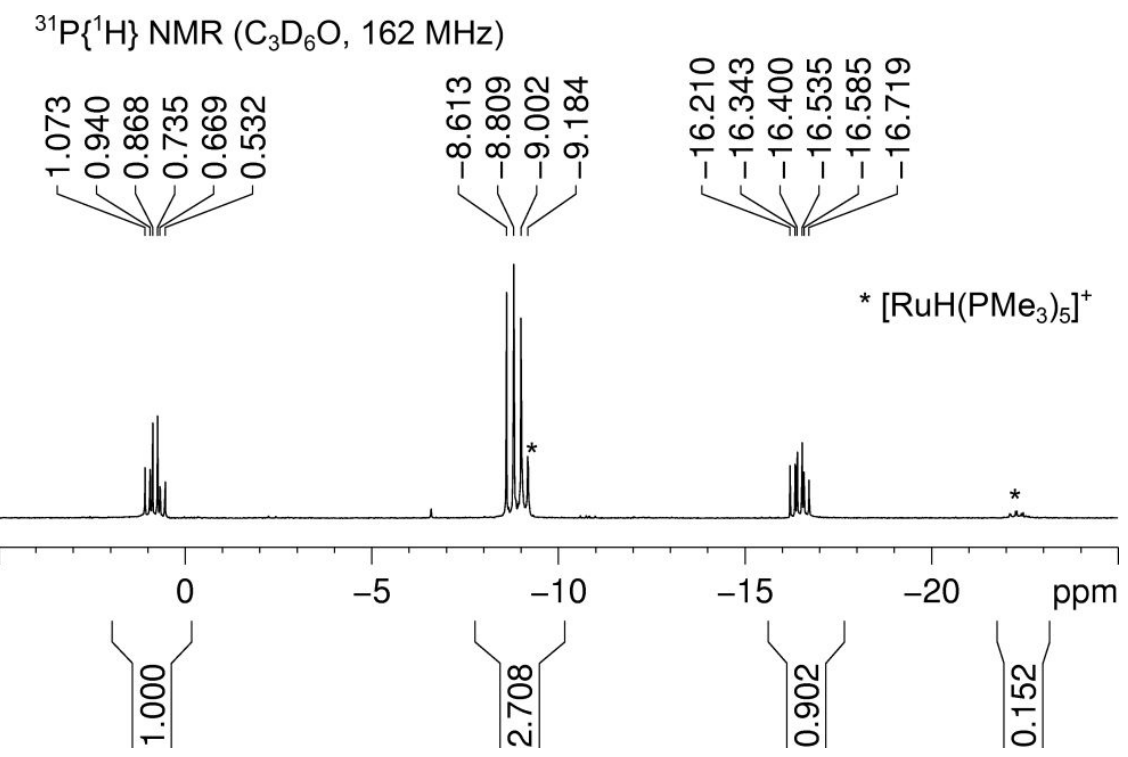

Figure S12. ${ }^{1} \mathrm{H}$ NMR spectrum for $\left[\mathrm{Ru}\left(\eta{ }^{3}-\mathrm{CF}_{3}-4-\mathrm{C}_{6} \mathrm{H}_{4} \mathrm{C} \equiv \mathrm{C}-\mathrm{C}=\mathrm{CH}\left(\mathrm{C}_{6} \mathrm{H}_{4}-4-\mathrm{CF}_{3}\right)\right)\left(\mathrm{PMe}_{3}\right)_{4}\right]+\mathrm{BPh}_{4}^{-} \mathbf{1 c}^{-\mathbf{B P h}_{4}}\left(\right.$ acetone- $d_{6}$, $400 \mathrm{MHz}$ )

\section{${ }^{1} \mathrm{H}$ NMR $\left(\mathrm{C}_{3} \mathrm{D}_{6} \mathrm{O}, 400 \mathrm{MHz}\right)$}

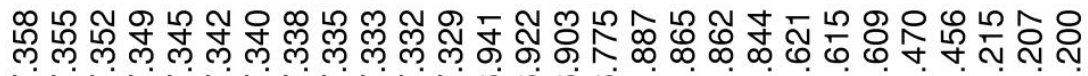

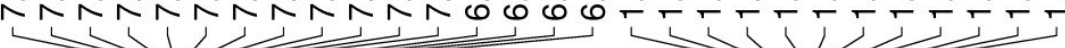

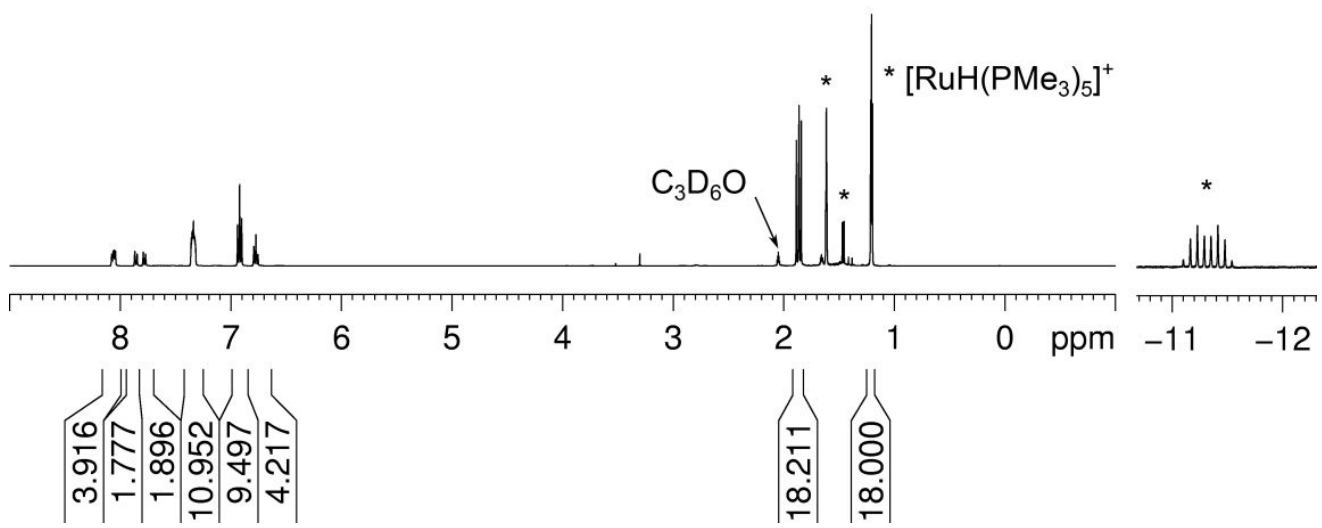


Figure S13. ${ }^{19} \mathrm{~F}$ NMR spectrum for $\left[\mathrm{Ru}\left(\eta^{3}-\mathrm{CF}_{3}-4-\mathrm{C}_{6} \mathrm{H}_{4} \mathrm{C} \equiv \mathrm{C}-\mathrm{C}=\mathrm{CH}\left(\mathrm{C}_{6} \mathrm{H}_{4}-4-\mathrm{CF}_{3}\right)\right)\left(\mathrm{PMe}_{3}\right)_{4}\right]^{+} \mathrm{BPh}_{4}^{-} \mathbf{1 c}-\mathbf{B P h}_{4}\left(\right.$ acetone- $d_{6}$, $565 \mathrm{MHz}$ )

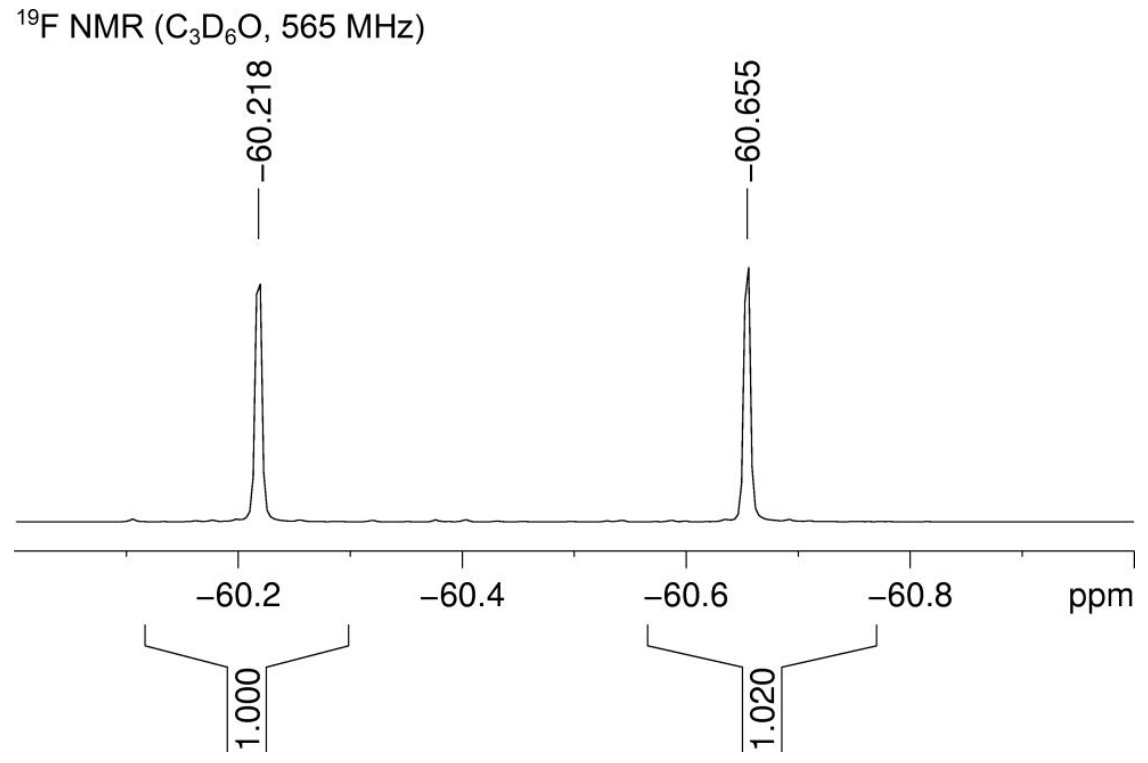

S2.5 $\quad\left[\mathrm{Ru}\left(\eta^{3}-\mathrm{CF}_{3}-4-\mathrm{C}_{6} \mathrm{H}_{4} \mathrm{C} \equiv \mathrm{C}-\mathrm{C}=\mathrm{CH}\left(\mathrm{C}_{6} \mathrm{H}_{4}-4-\mathrm{CF}_{3}\right)\right)\left(\mathrm{PMe}_{3}\right)_{4}\right]^{+} \mathrm{BF}_{4}^{-} \mathrm{IC}^{-}-\mathrm{BF}_{4}$.

Figure S14. ${ }^{31} \mathrm{P}\left\{{ }_{1} \mathrm{H}\right\} \quad \mathrm{NMR}$ spectrum for $\left[\mathrm{Ru}\left(\eta^{3}-\mathrm{CF}_{3}-4-\mathrm{C}_{6} \mathrm{H}_{4} \mathrm{C} \equiv \mathrm{C}-\mathrm{C}=\mathrm{CH}\left(\mathrm{C}_{6} \mathrm{H}_{4}-4-\mathrm{CF}_{3}\right)\right)\left(\mathrm{PMe}_{3}\right)_{4}\right]^{+} \quad \mathrm{BF}_{4}^{-} \quad \mathbf{1 C}^{-}-\mathrm{BF}_{4}$ (acetone- $d_{6}, 162 \mathrm{MHz}$ )

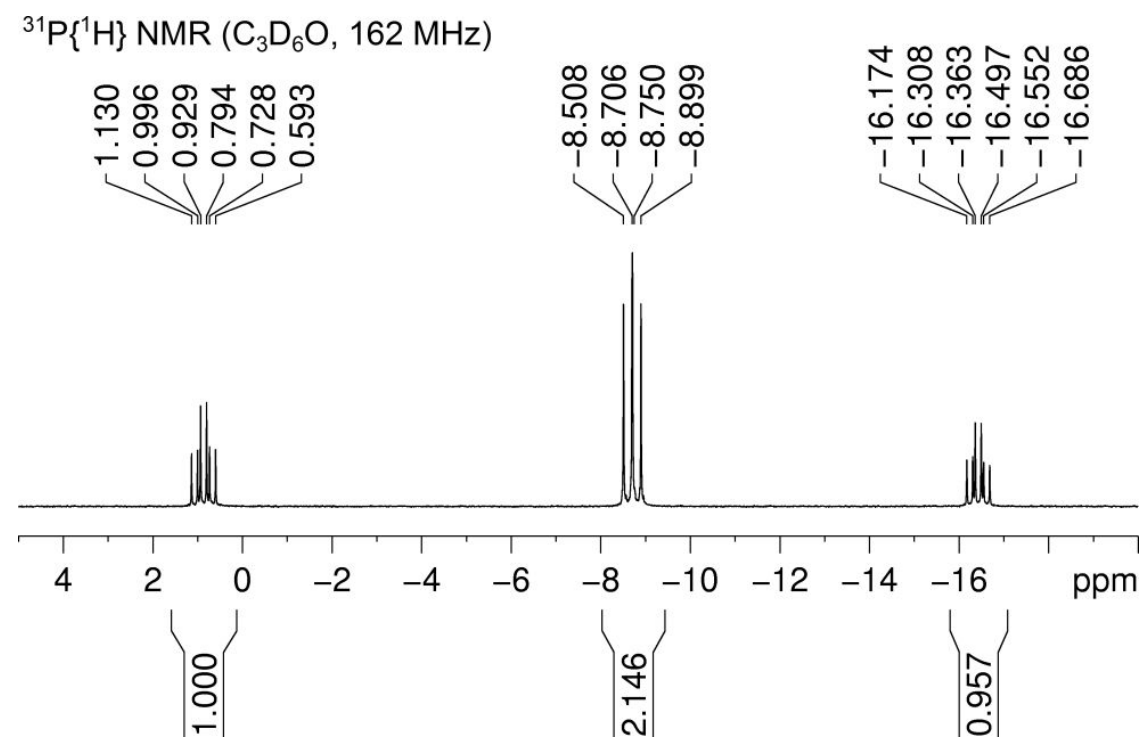


Figure S15. ${ }^{1} \mathrm{H}$ NMR spectrum for $\left[\mathrm{Ru}\left(\eta^{3}-\mathrm{CF}_{3}-4-\mathrm{C}_{6} \mathrm{H}_{4} \mathrm{C} \equiv \mathrm{C}-\mathrm{C}=\mathrm{CH}\left(\mathrm{C}_{6} \mathrm{H}_{4}-4-\mathrm{CF}_{3}\right)\right)\left(\mathrm{PMe}_{3}\right)_{4}\right]^{+} \mathrm{BF}_{4}^{-} \mathbf{1 c}^{-} \mathbf{B F}_{4}$ (acetone- $d_{6}$, $400 \mathrm{MHz}$ )

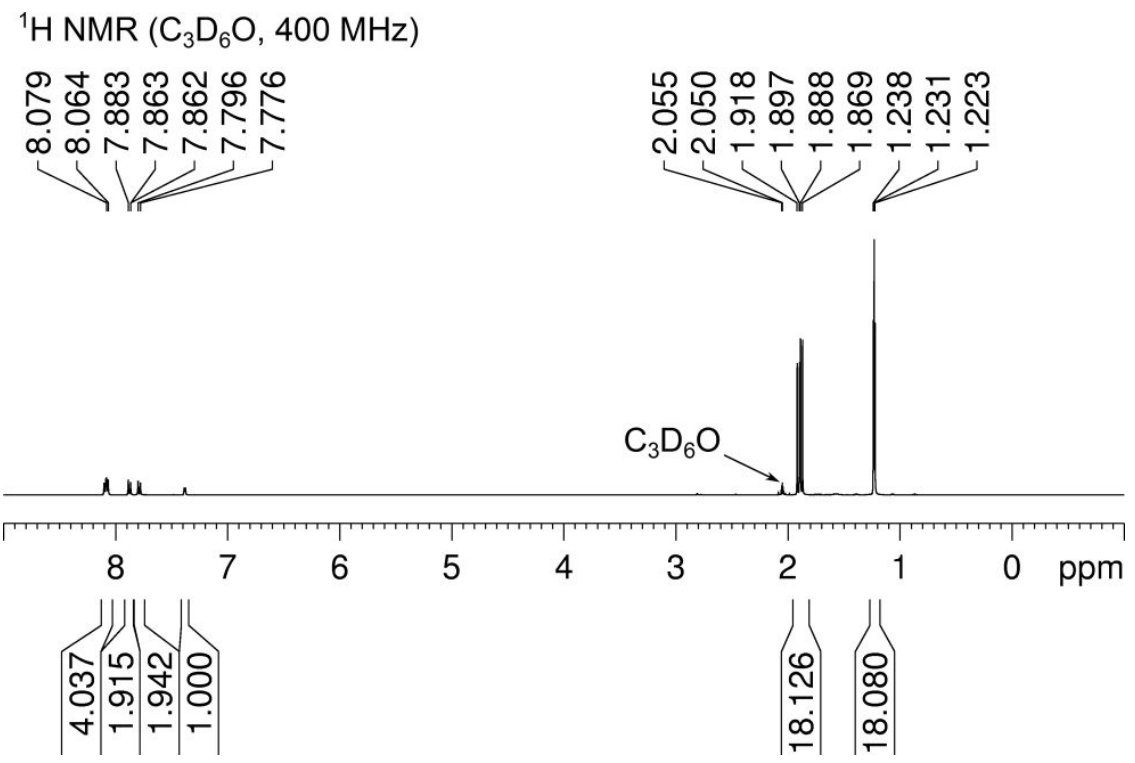

Figure S16. ${ }^{13} \mathrm{C}\left\{{ }^{1} \mathrm{H}\right\} \quad \mathrm{NMR}$ spectrum for $\left[\mathrm{Ru}\left(\eta^{3}-\mathrm{CF}_{3}-4-\mathrm{C}_{6} \mathrm{H}_{4} \mathrm{C} \equiv \mathrm{C}-\mathrm{C}=\mathrm{CH}\left(\mathrm{C}_{6} \mathrm{H}_{4}-4-\mathrm{CF}_{3}\right)\right)\left(\mathrm{PMe}_{3}\right)_{4}\right]^{+} \quad \mathrm{BF}_{4}^{-} \quad \mathbf{1 C}^{-} \mathrm{BF}_{4}$ (acetone- $d_{6}, 101 \mathrm{MHz}$ )

${ }^{13} \mathrm{C}\left\{{ }^{1} \mathrm{H}\right\} \operatorname{NMR}\left(\mathrm{C}_{3} \mathrm{D}_{6} \mathrm{O}, 101 \mathrm{MHz}\right)$

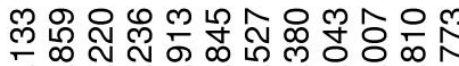
ปั่

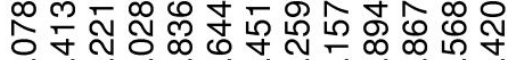

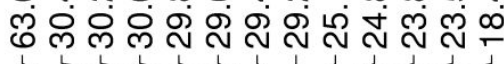
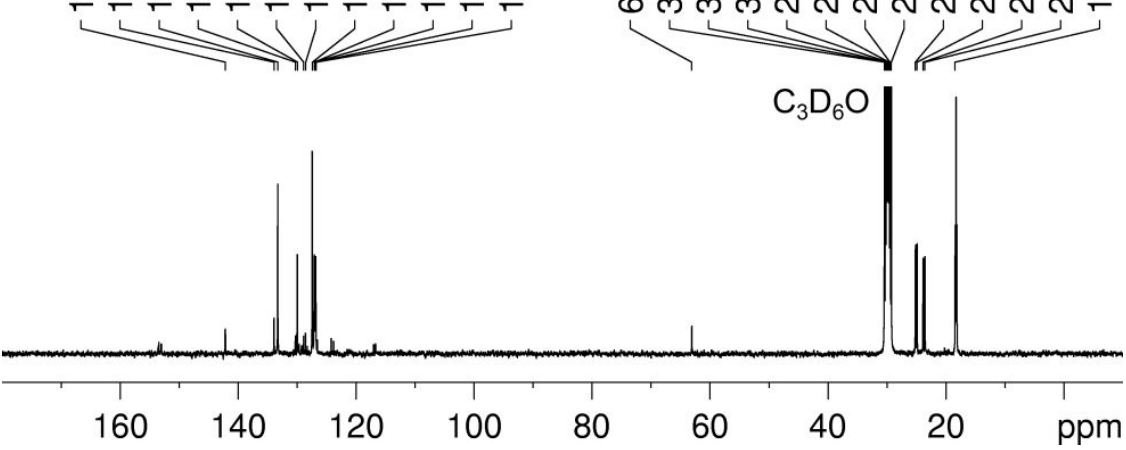
Figure S17. ${ }^{19} \mathrm{~F}$ NMR spectrum for $\left[\mathrm{Ru}\left(\eta^{3}-\mathrm{CF}_{3}-4-\mathrm{C}_{6} \mathrm{H}_{4} \mathrm{C} \equiv \mathrm{C}-\mathrm{C}=\mathrm{CH}\left(\mathrm{C}_{6} \mathrm{H}_{4}-4-\mathrm{CF}_{3}\right)\right)\left(\mathrm{PMe}_{3}\right)_{4}\right]^{+} \mathrm{BF}_{4}^{-}$1c- $\mathbf{B F}_{4}$ (acetone- $d_{6}$, $376 \mathrm{MHz}$ )

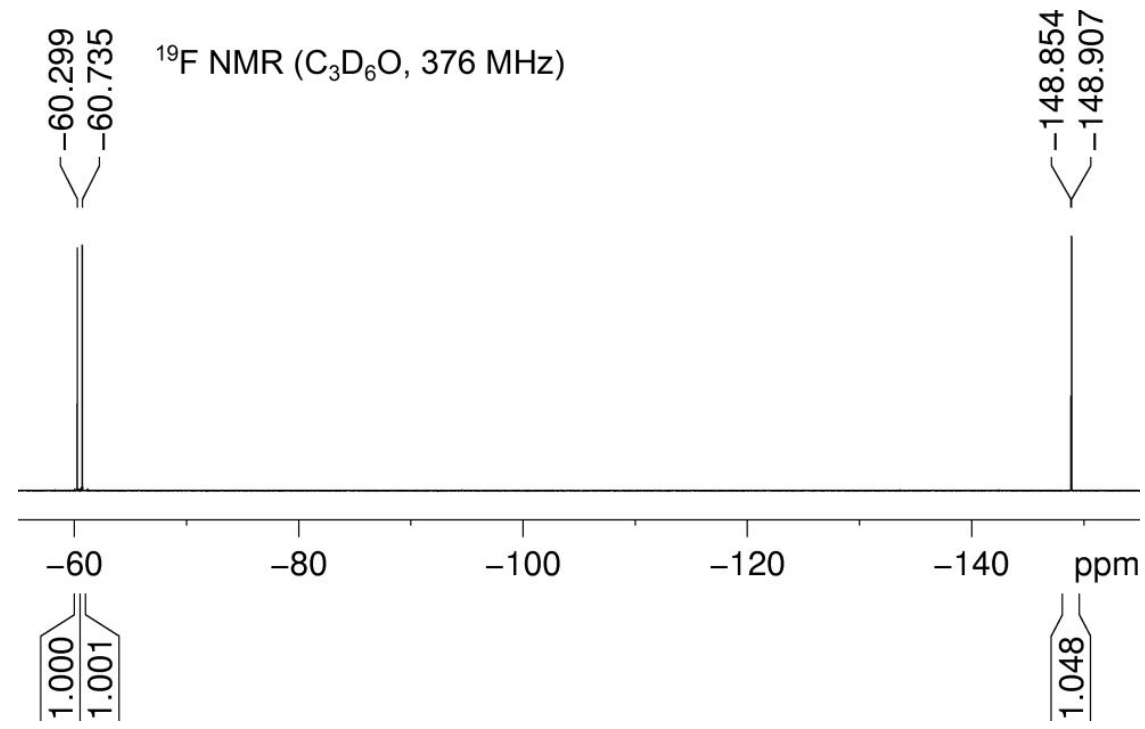

S2.6 $\left[\mathrm{Ru}\left(\mathrm{C}\left(\mathrm{C} \equiv \mathrm{CC}_{6} \mathrm{H}_{4}-4-\mathrm{OMe}\right)=\mathrm{CH}\left(\mathrm{C}_{6} \mathrm{H}_{3}-4-\mathrm{OMe}\right)\left(\mathrm{PMe}_{3}\right)_{4}\right] 2 \mathrm{a}\right.$.

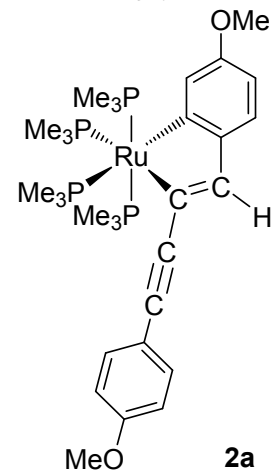

Figure S18. ${ }^{31} \mathrm{P}\left\{{ }^{1} \mathrm{H}\right\}$ NMR spectrum for $\left[\mathrm{Ru}\left(\mathrm{C}\left(\mathrm{C} \equiv \mathrm{CC}_{6} \mathrm{H}_{4}-4-\mathrm{OMe}\right)=\mathrm{CH}\left(\mathrm{C}_{6} \mathrm{H}_{3}-4-\mathrm{OMe}\right)\left(\mathrm{PMe}_{3}\right)_{4}\right]\right.$ 2a (tetrahydrofuran- $d_{8}$, $162 \mathrm{MHz}$ )

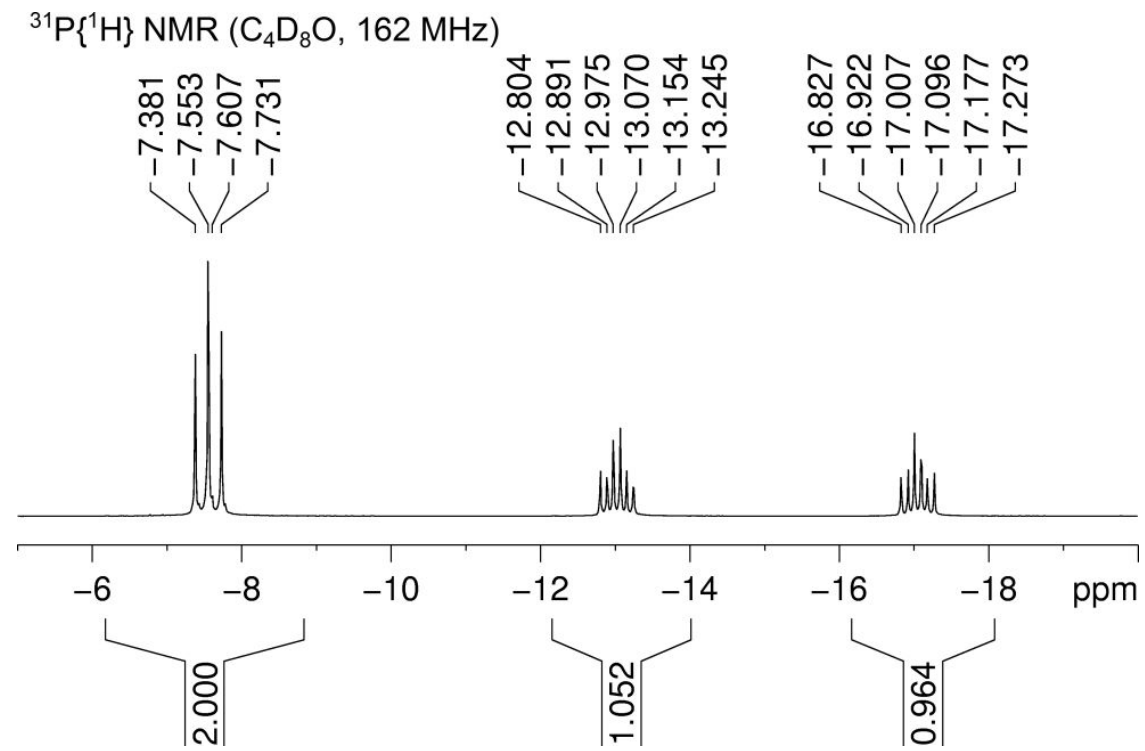


Figure S19. ${ }^{1} \mathrm{H}$ NMR spectrum for $\left[\mathrm{Ru}\left(\mathrm{C}\left(\mathrm{C} \equiv \mathrm{CC}_{6} \mathrm{H}_{4}-4-\mathrm{OMe}\right)=\mathrm{CH}\left(\mathrm{C}_{6} \mathrm{H}_{3}-4-\mathrm{OMe}\right)\left(\mathrm{PMe}_{3}\right)_{4}\right]\right.$ 2a (tetrahydrofuran- $d_{8}$, $400 \mathrm{MHz})$

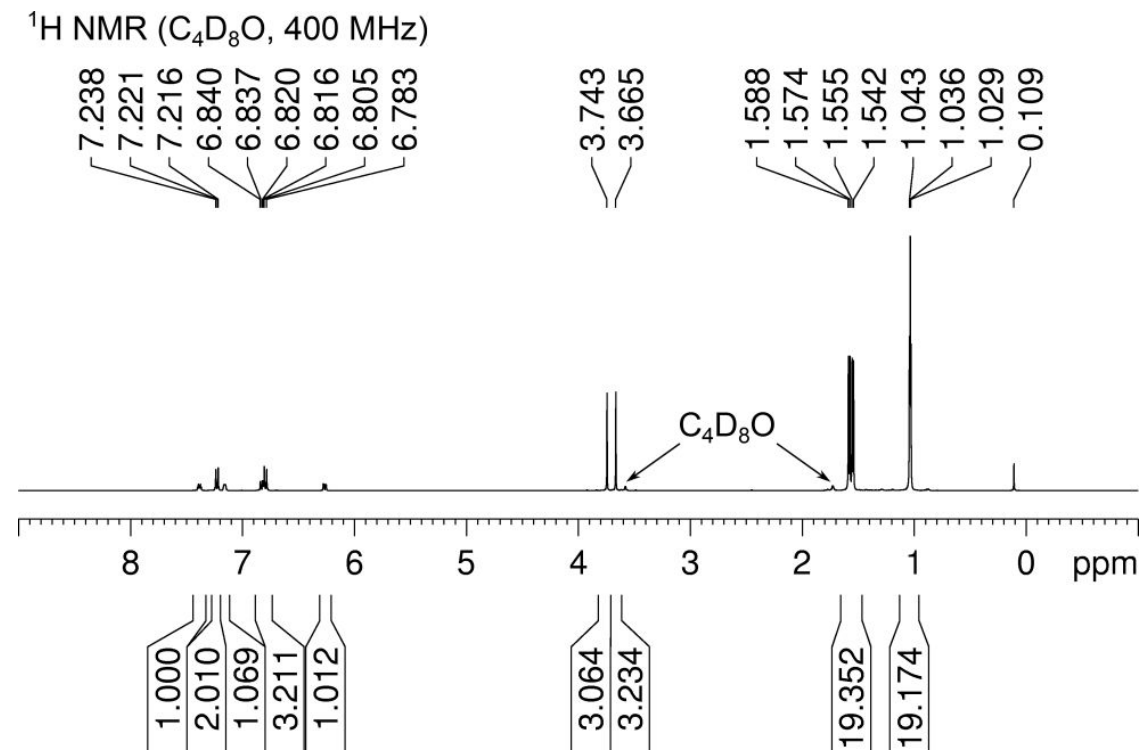

Figure S2o. ${ }^{13} \mathrm{C}\left\{{ }^{1} \mathrm{H}\right\} \quad \mathrm{NMR}$ spectrum for $\left[\mathrm{Ru}\left(\mathrm{C}\left(\mathrm{C} \equiv \mathrm{CC}_{6} \mathrm{H}_{4}-4-\mathrm{OMe}\right)=\mathrm{CH}\left(\mathrm{C}_{6} \mathrm{H}_{3}-4-\mathrm{OMe}\right)\left(\mathrm{PMe}_{3}\right)_{4}\right] \quad\right.$ 2a (tetrahydrofuran- $d_{8}, 101 \mathrm{MHz}$ )

${ }^{13} \mathrm{C}\left\{{ }^{1} \mathrm{H}\right\} \operatorname{NMR}\left(\mathrm{C}_{4} \mathrm{D}_{8} \mathrm{O}, 101 \mathrm{MHz}\right)$

유ㅇㅠㅝ 寸

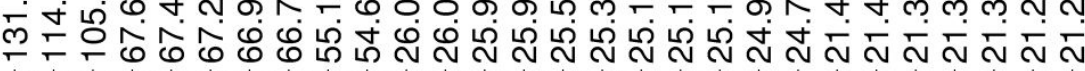

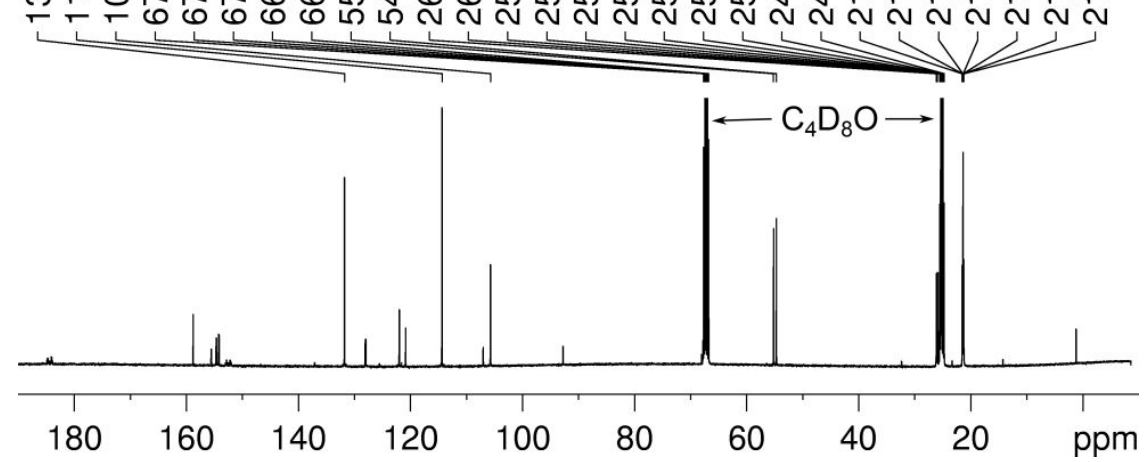


Figure S21. HRMS for $\left[\mathrm{Ru}\left(\mathrm{C}\left(\mathrm{C} \equiv \mathrm{CC}_{6} \mathrm{H}_{4}-4-\mathrm{OMe}\right)=\mathrm{CH}\left(\mathrm{C}_{6} \mathrm{H}_{3}-4-\mathrm{OMe}\right)\left(\mathrm{PMe}_{3}\right)_{4}\right] 2 \mathrm{a}\right.$ (acetonitrile, ESI) 46-94-LHL_Pos_Full \#42-53 RT: 1.18-1.50 AV: 12 NL: 2.90E7

T: FTMS + p NSI Full ms [100.00-2000.00]

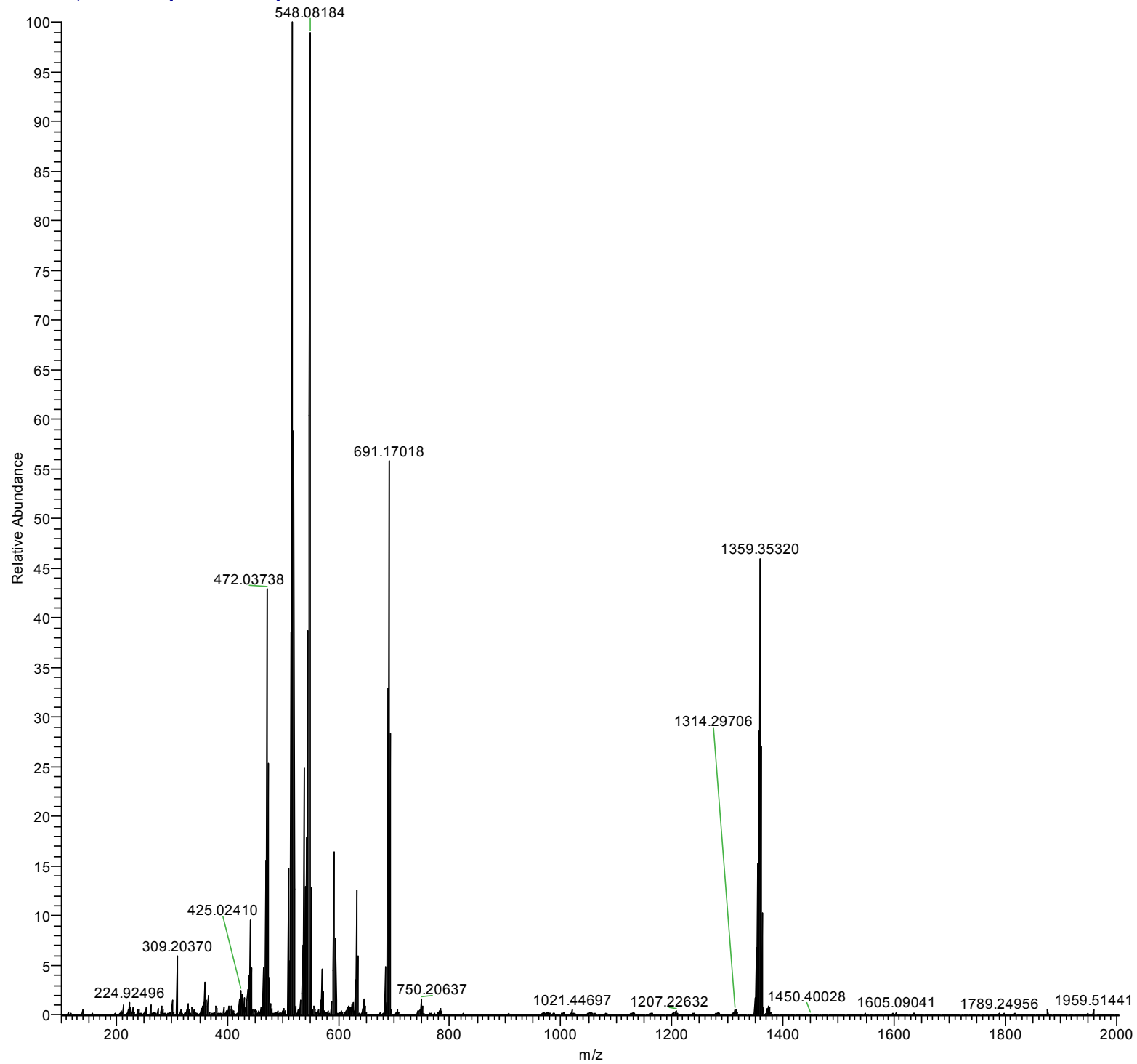


Figure S22. HRMS for $\left[\mathrm{Ru}\left(\mathrm{C}\left(\mathrm{C} \equiv \mathrm{CC}_{6} \mathrm{H}_{4}-4-\mathrm{OMe}\right)=\mathrm{CH}\left(\mathrm{C}_{6} \mathrm{H}_{3}-4-\mathrm{OMe}\right)\left(\mathrm{PMe}_{3}\right)_{4}\right]\right.$ 2a (expansion, acetonitrile, ESI $)$

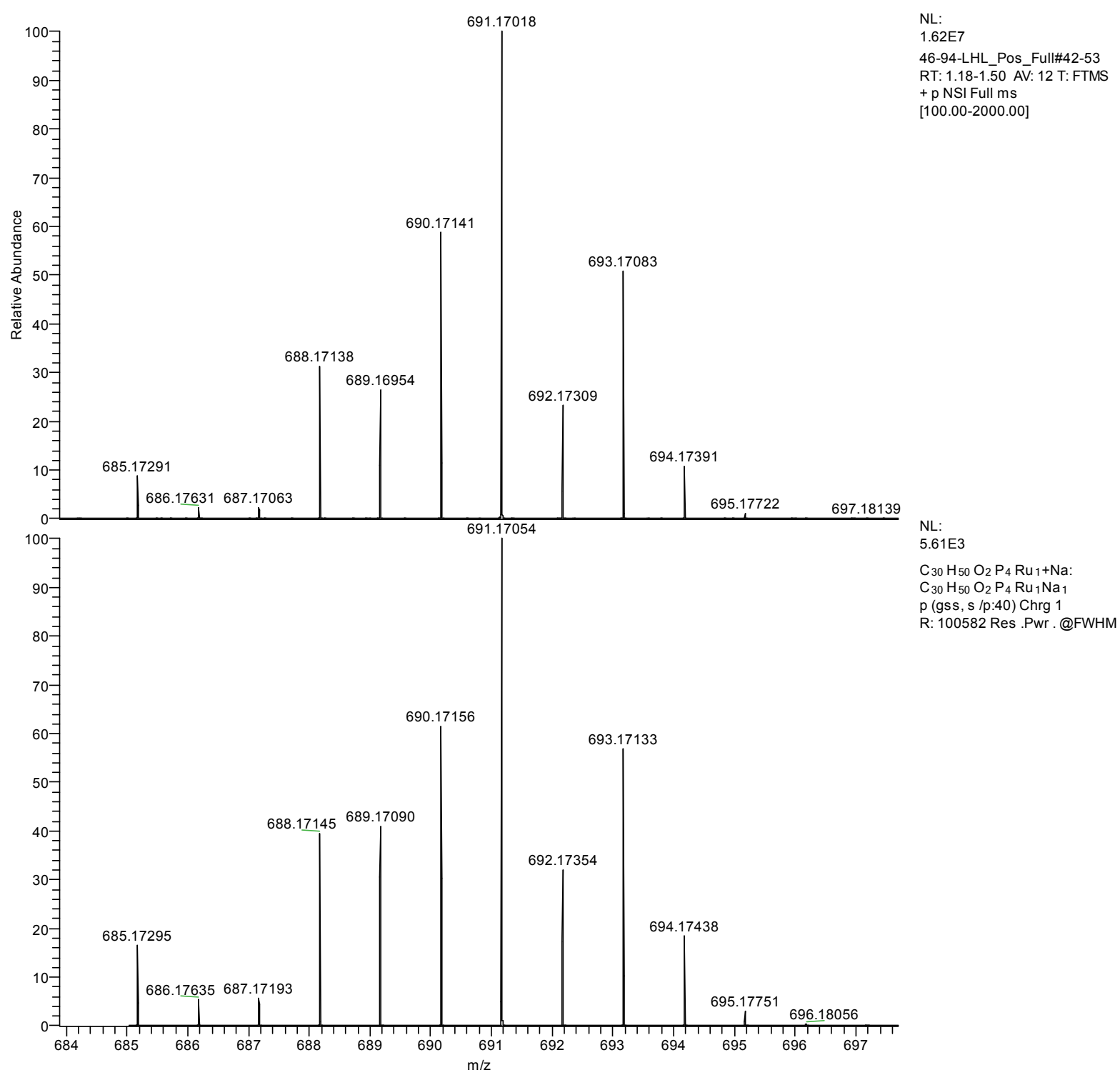




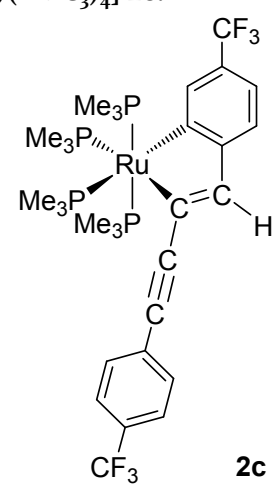

Figure S23. ${ }^{31} \mathrm{P}\left\{{ }^{1} \mathrm{H}\right\}$ NMR spectrum for $\left[\mathrm{Ru}\left(\mathrm{CF}_{3}-4-\mathrm{C}_{6} \mathrm{H}_{4} \mathrm{C} \equiv \mathrm{C}-\mathrm{C}=\mathrm{CH}\left(\mathrm{C}_{6} \mathrm{H}_{3}-4-\mathrm{CF}_{3}\right)\left(\mathrm{PMe}_{3}\right)_{4}\right]\right.$ 2c (tetrahydrofuran- $d_{8}$, $243 \mathrm{MHz}$ )

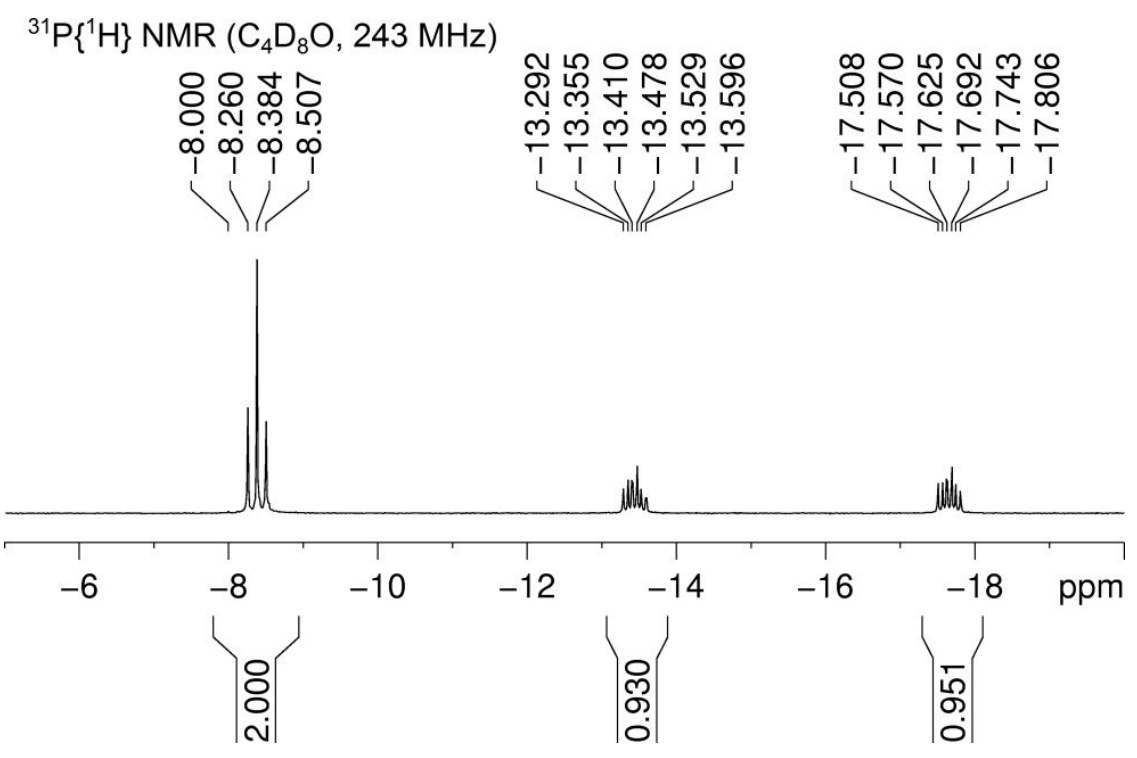

Figure S24. ${ }^{1} \mathrm{H}$ NMR spectrum for $\left[\mathrm{Ru}\left(\mathrm{CF}_{3}-4-\mathrm{C}_{6} \mathrm{H}_{4} \mathrm{C} \equiv \mathrm{C}-\mathrm{C}=\mathrm{CH}\left(\mathrm{C}_{6} \mathrm{H}_{3}-4-\mathrm{CF}_{3}\right)\left(\mathrm{PMe}_{3}\right)_{4}\right]\right.$ 2c (tetrahydrofuran- $d_{8}$, $600 \mathrm{MHz}$ )

${ }^{1} \mathrm{H}$ NMR $\left(\mathrm{C}_{4} \mathrm{D}_{8} \mathrm{O}, 600 \mathrm{MHz}\right)$

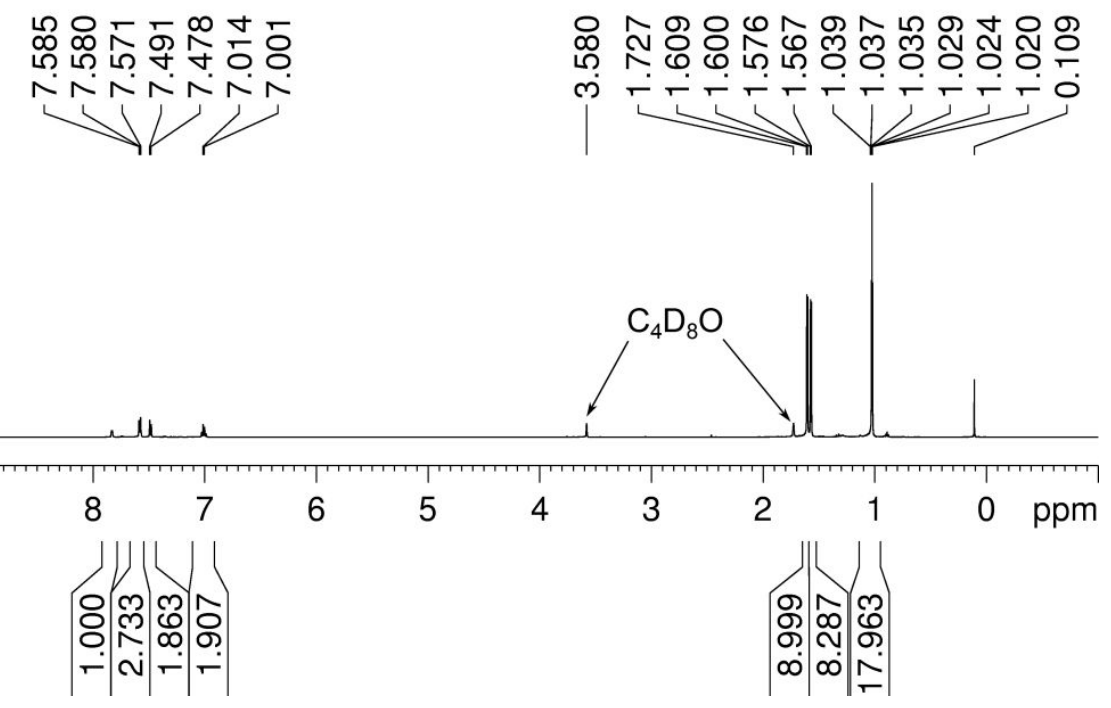


Figure S25. ${ }^{13} \mathrm{C}\left\{{ }^{1} \mathrm{H}\right\}$ NMR spectrum for $\left[\mathrm{Ru}\left(\mathrm{CF}_{3}-4-\mathrm{C}_{6} \mathrm{H}_{4} \mathrm{C} \equiv \mathrm{C}-\mathrm{C}=\mathrm{CH}\left(\mathrm{C}_{6} \mathrm{H}_{3}-4-\mathrm{CF}_{3}\right)\left(\mathrm{PMe}_{3}\right)_{4}\right]\right.$ 2c (tetrahydrofuran- $d_{8}$, $151 \mathrm{MHz}$ )

$$
{ }^{13} \mathrm{C}\left\{{ }^{1} \mathrm{H}\right\} \operatorname{NMR}\left(\mathrm{C}_{4} \mathrm{D}_{8} \mathrm{O}, 151 \mathrm{MHz}\right)
$$

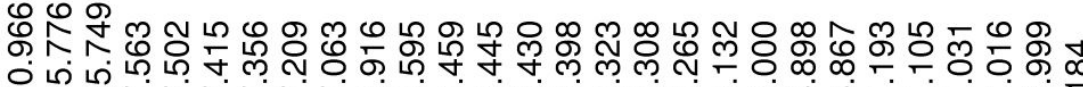

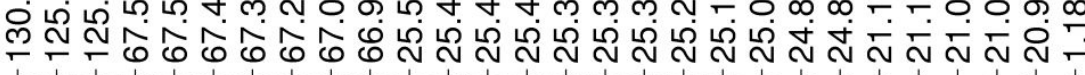

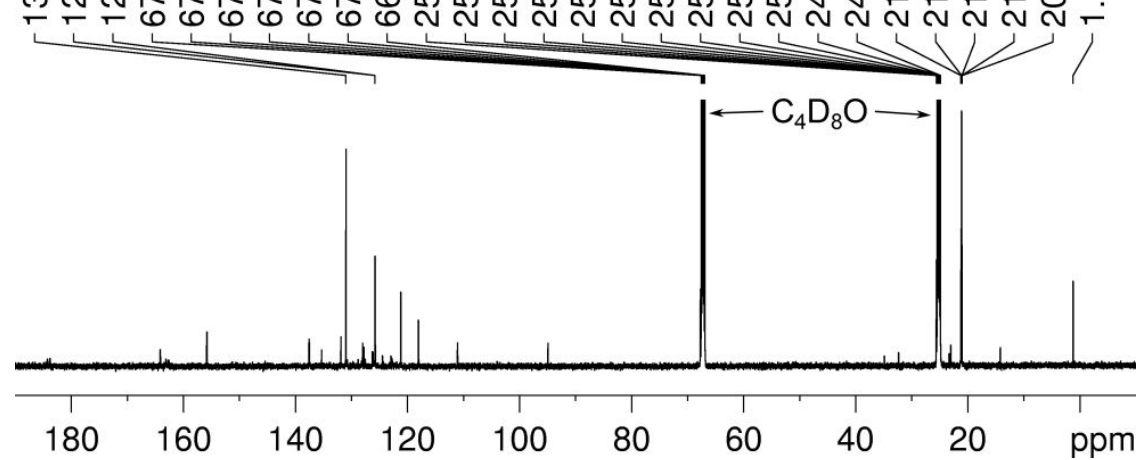

Figure S26. ${ }^{19} \mathrm{~F}$ NMR spectrum for $\left[\mathrm{Ru}\left(\mathrm{CF}_{3}-4-\mathrm{C}_{6} \mathrm{H}_{4} \mathrm{C} \equiv \mathrm{C}-\mathrm{C}=\mathrm{CH}\left(\mathrm{C}_{6} \mathrm{H}_{3}-4-\mathrm{CF}_{3}\right)\left(\mathrm{PMe}_{3}\right)_{4}\right]\right.$ 2c (tetrahydrofuran- $d_{8}$, $565 \mathrm{MHz})$

${ }^{19} \mathrm{~F}$ NMR $\left(\mathrm{C}_{4} \mathrm{D}_{8} \mathrm{O}, 565 \mathrm{MHz}\right)$

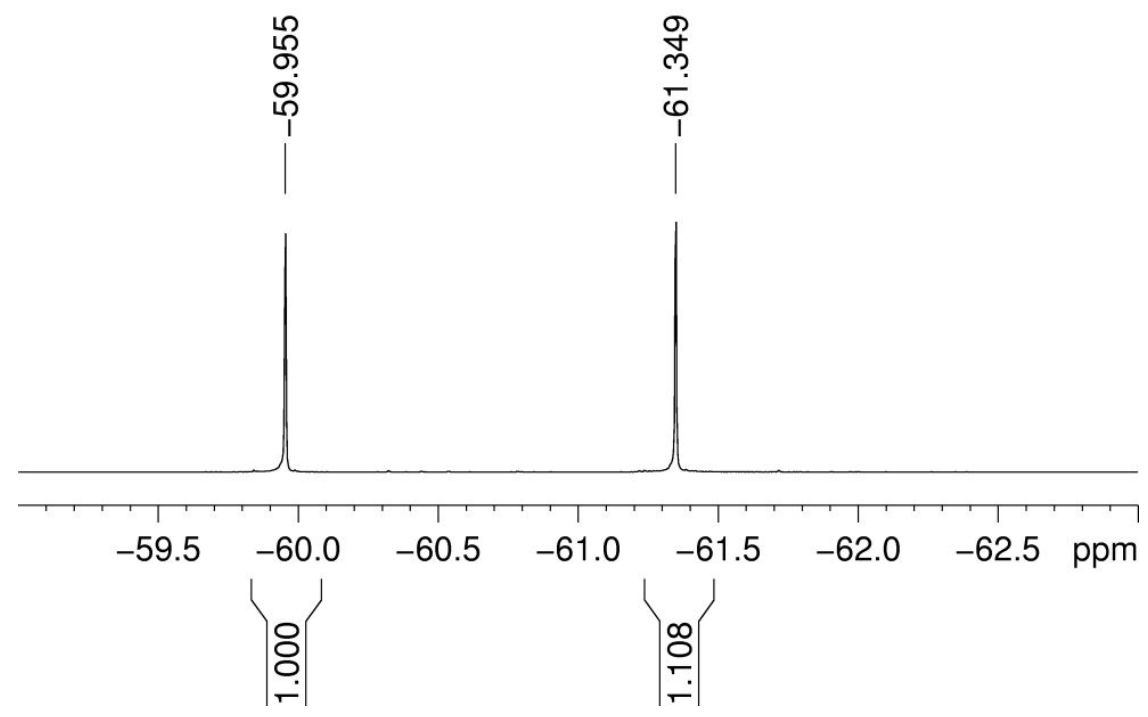


Figure S27. HRMS for $\left[\mathrm{Ru}\left(\mathrm{CF}_{3}-4-\mathrm{C}_{6} \mathrm{H}_{4} \mathrm{C} \equiv \mathrm{C}-\mathrm{C}=\mathrm{CH}\left(\mathrm{C}_{6} \mathrm{H}_{3}-4-\mathrm{CF}_{3}\right)\left(\mathrm{PMe}_{3}\right)_{4}\right] 2 \mathrm{c}\right.$ (acetonitrile, ESI) 47-31-LHL_Pos_Full \#7-12 RT: 0.20-0.34 AV: 6 NL: $2.41 \mathrm{E} 8$

T: FTMS + p NSI Full ms [100.00-2000.00]

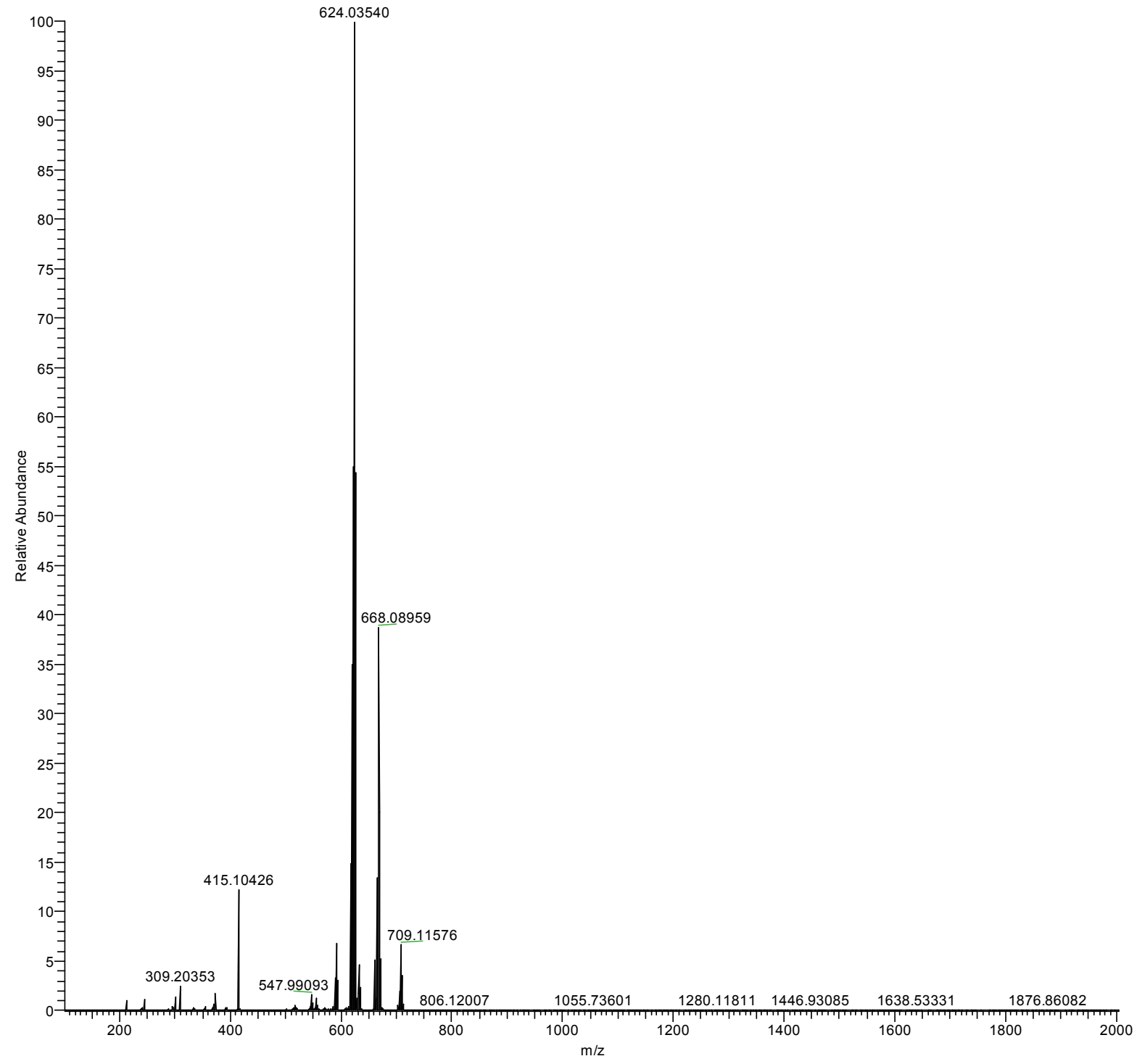


Figure S28. HRMS for $\left[\mathrm{Ru}\left(\mathrm{CF}_{3}-4-\mathrm{C}_{6} \mathrm{H}_{4} \mathrm{C} \equiv \mathrm{C}-\mathrm{C}=\mathrm{CH}\left(\mathrm{C}_{6} \mathrm{H}_{3}-4-\mathrm{CF}_{3}\right)\left(\mathrm{PMe}_{3}\right)_{4}\right] 2 \mathrm{c}\right.$ (expansion, acetonitrile, ESI)

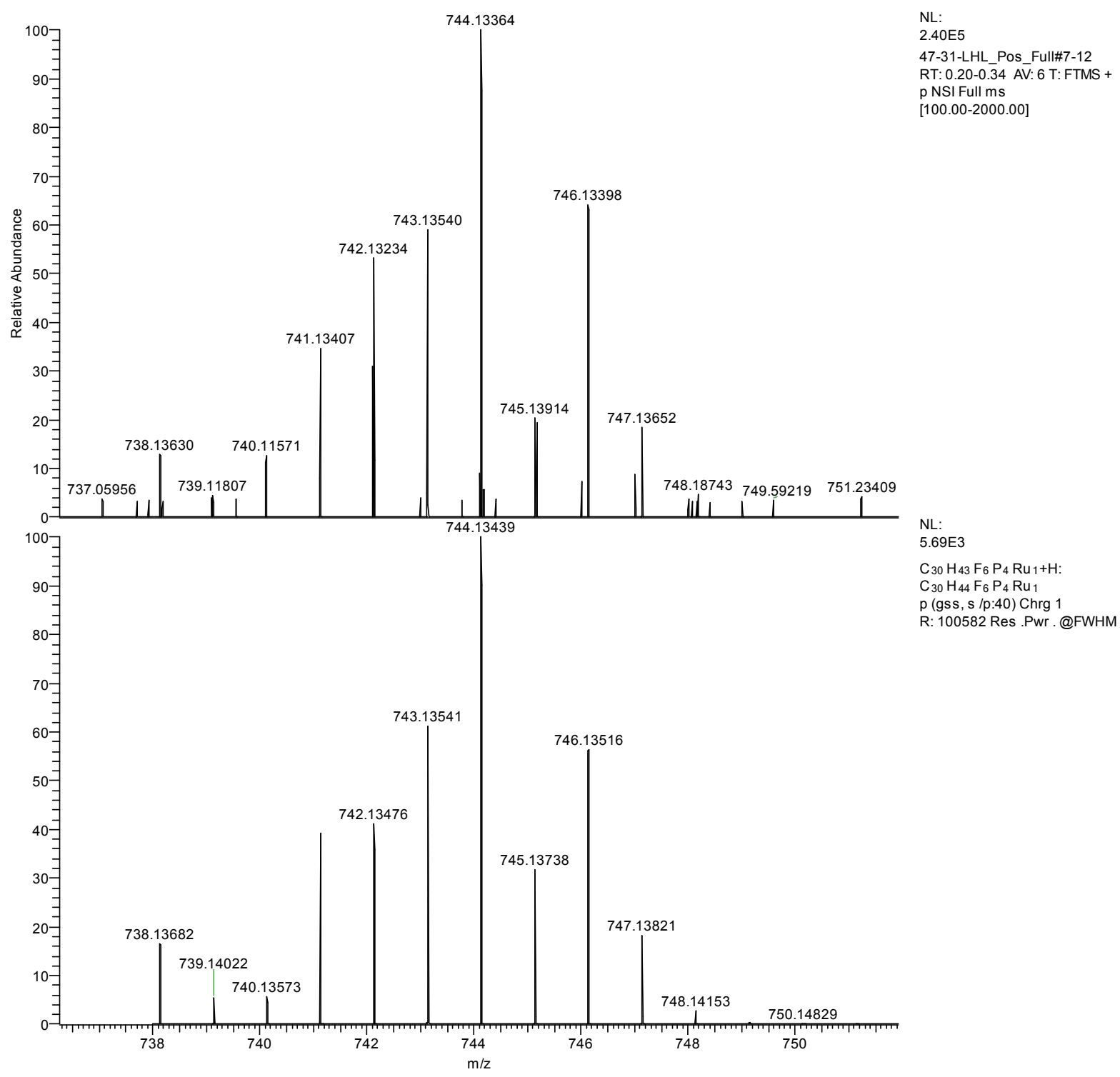


S2.8 cis/trans- $\left[\mathrm{Ru}\left(\mathrm{C} \equiv \mathrm{CC}_{6} \mathrm{H}_{4}-4-\mathrm{OMe}\right)_{2}\left(\mathrm{PMe}_{3}\right)_{4}\right]$ 3a.

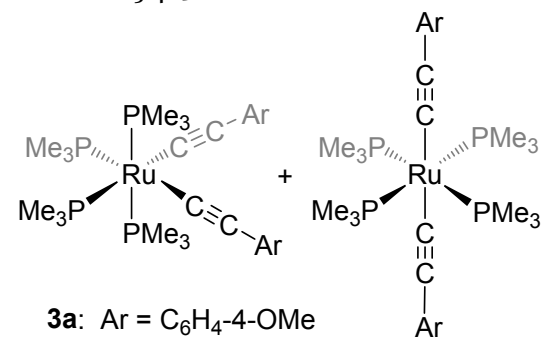

Figure S29. ${ }^{31} \mathrm{P}\left\{{ }^{1} \mathrm{H}\right\}$ NMR spectrum for cis/trans- $\left[\mathrm{Ru}\left(\mathrm{C} \equiv \mathrm{CC}_{6} \mathrm{H}_{4}-4-\mathrm{OMe}\right)_{2}\left(\mathrm{PMe}_{3}\right)_{4}\right]$ 3a (tetrahydrofuran- $\left.d_{8}, 162 \mathrm{MHz}\right)$ ${ }^{31} \mathrm{P}\left\{{ }^{1} \mathrm{H}\right\} \operatorname{NMR}\left(\mathrm{C}_{4} \mathrm{D}_{8} \mathrm{O}, 162 \mathrm{MHz}\right)$
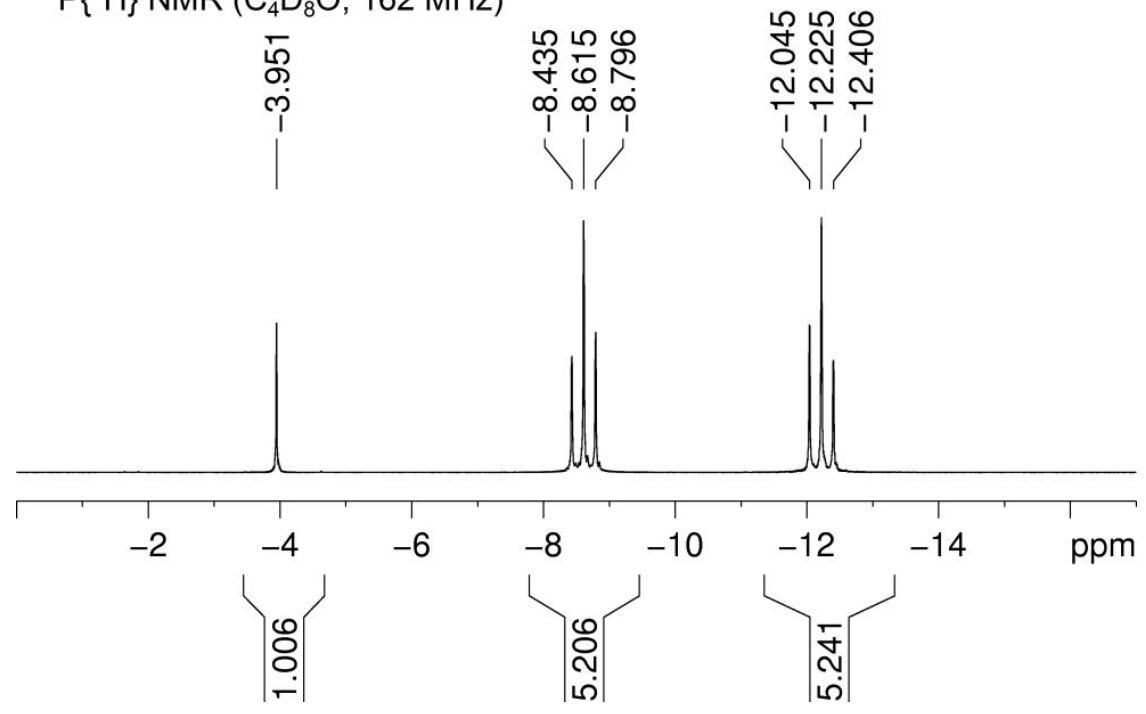

Figure S3o. ${ }^{1} \mathrm{H}$ NMR spectrum for cis/trans- $\left[\mathrm{Ru}\left(\mathrm{C} \equiv \mathrm{CC}_{6} \mathrm{H}_{4}-4-\mathrm{OMe}\right)_{2}\left(\mathrm{PMe}_{3}\right)_{4}\right]$ 3a (tetrahydrofuran- $\left.d_{8}, 400 \mathrm{MHz}\right)$ ${ }^{1} \mathrm{H}$ NMR $\left(\mathrm{C}_{4} \mathrm{D}_{8} \mathrm{O}, 400 \mathrm{MHz}\right)$

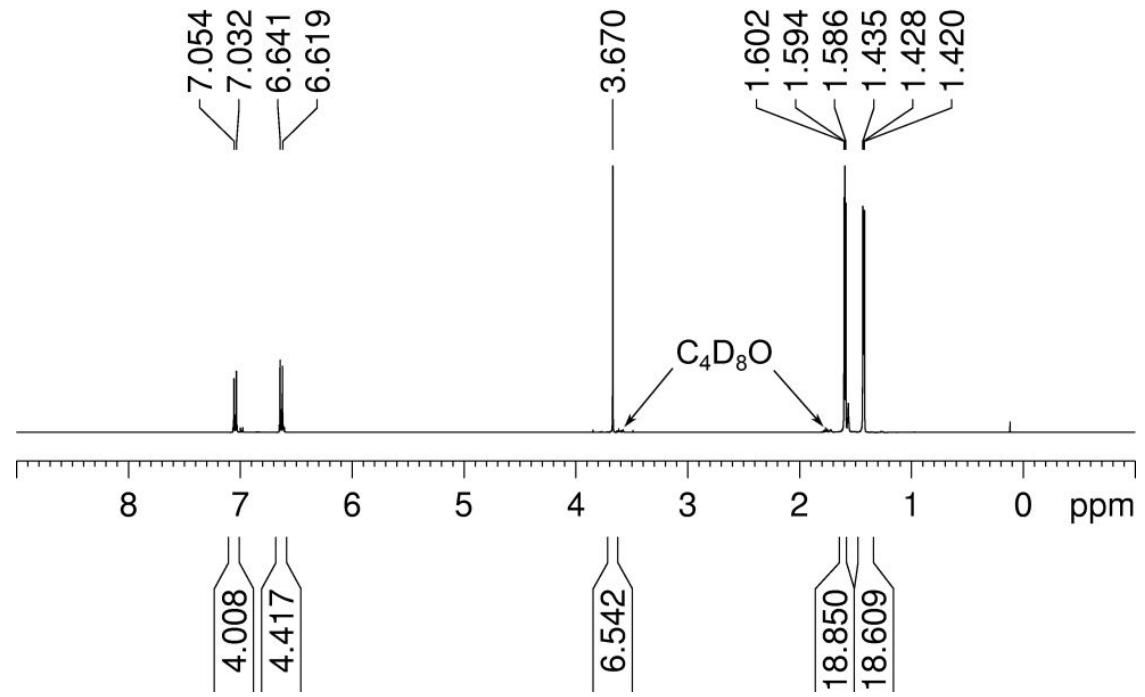


Figure S31. ${ }^{13} \mathrm{C}\left\{{ }_{1} \mathrm{H}\right\}$ NMR spectrum for cis/trans- $\left[\mathrm{Ru}\left(\mathrm{C} \equiv \mathrm{CC}_{6} \mathrm{H}_{4}-4-\mathrm{OMe}\right)_{2}\left(\mathrm{PMe}_{3}\right)_{4}\right]$ 3a (tetrahydrofuran- $\left.d_{8}, 101 \mathrm{MHz}\right)$ ${ }^{13} \mathrm{C}\left\{{ }^{1} \mathrm{H}\right\} \operatorname{NMR}\left(\mathrm{C}_{4} \mathrm{D}_{8} \mathrm{O}, 101 \mathrm{MHz}\right)$

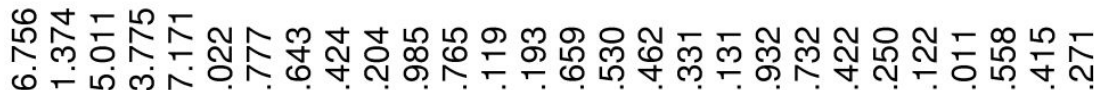

மํำ

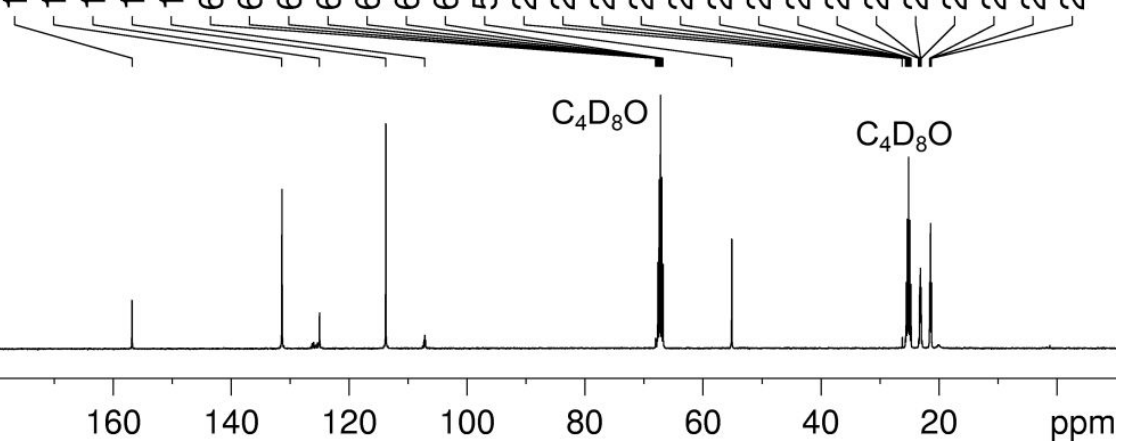

Figure $S_{32}$. HRMS for cis/trans-[Ru(C $\left.\left.\equiv \mathrm{CC}_{6} \mathrm{H}_{4}-4-\mathrm{OMe}\right)_{2}\left(\mathrm{PMe}_{3}\right)_{4}\right]$ 3a (acetonitrile, ESI)

43-87-LHL_Pos_Full \#6-18 RT: 0.30-0.97 AV: 13 NL: $3.99 E 7$

T: FTMS + p NSI Full ms [100.00-2000.00]

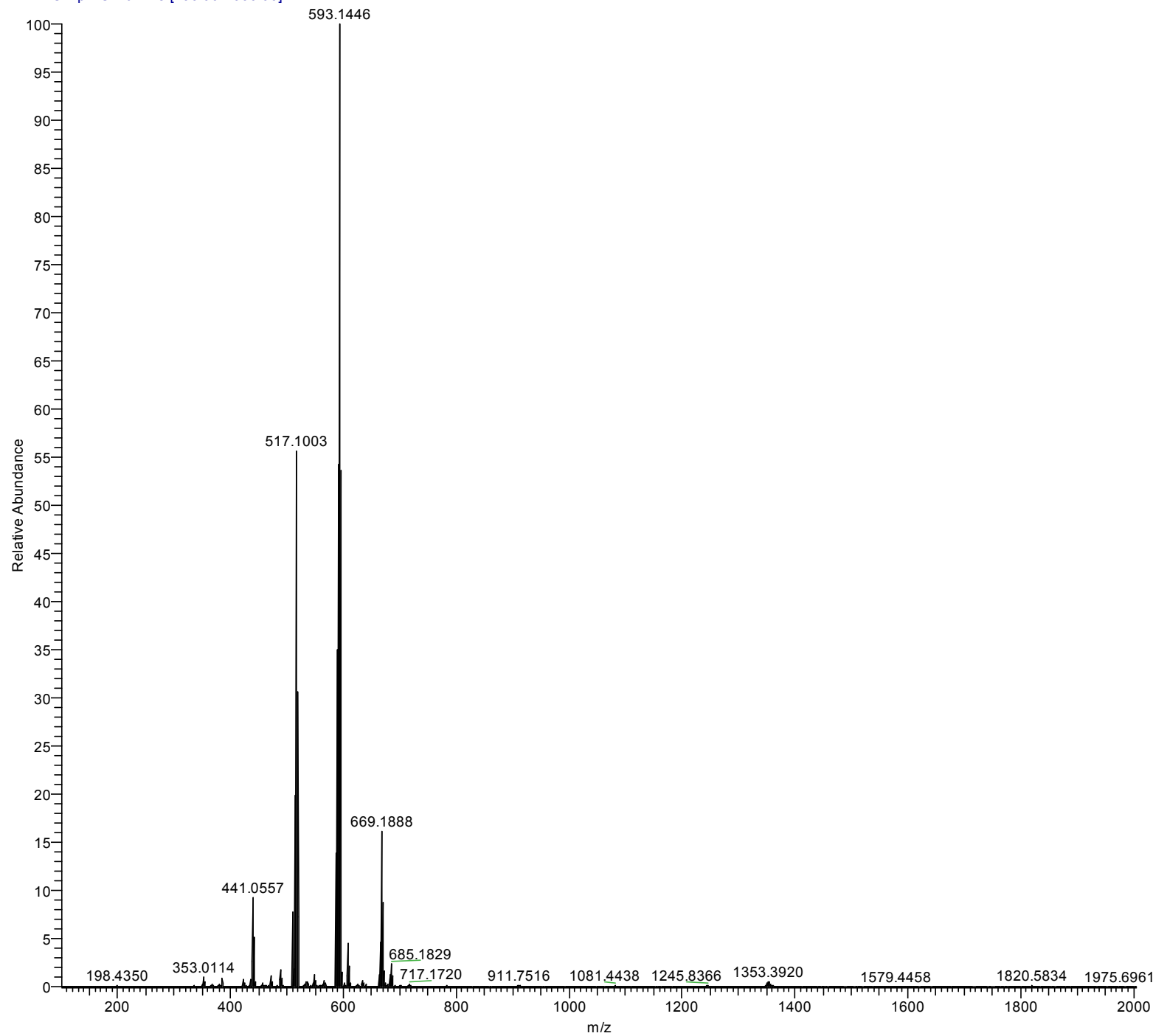


Figure S33. HRMS for cis/trans- $\left[\mathrm{Ru}\left(\mathrm{C} \equiv \mathrm{CC}_{6} \mathrm{H}_{4}-4-\mathrm{OMe}\right)_{2}\left(\mathrm{PMe}_{3}\right)_{4}\right]$ 3a (expansion, acetonitrile, ESI)

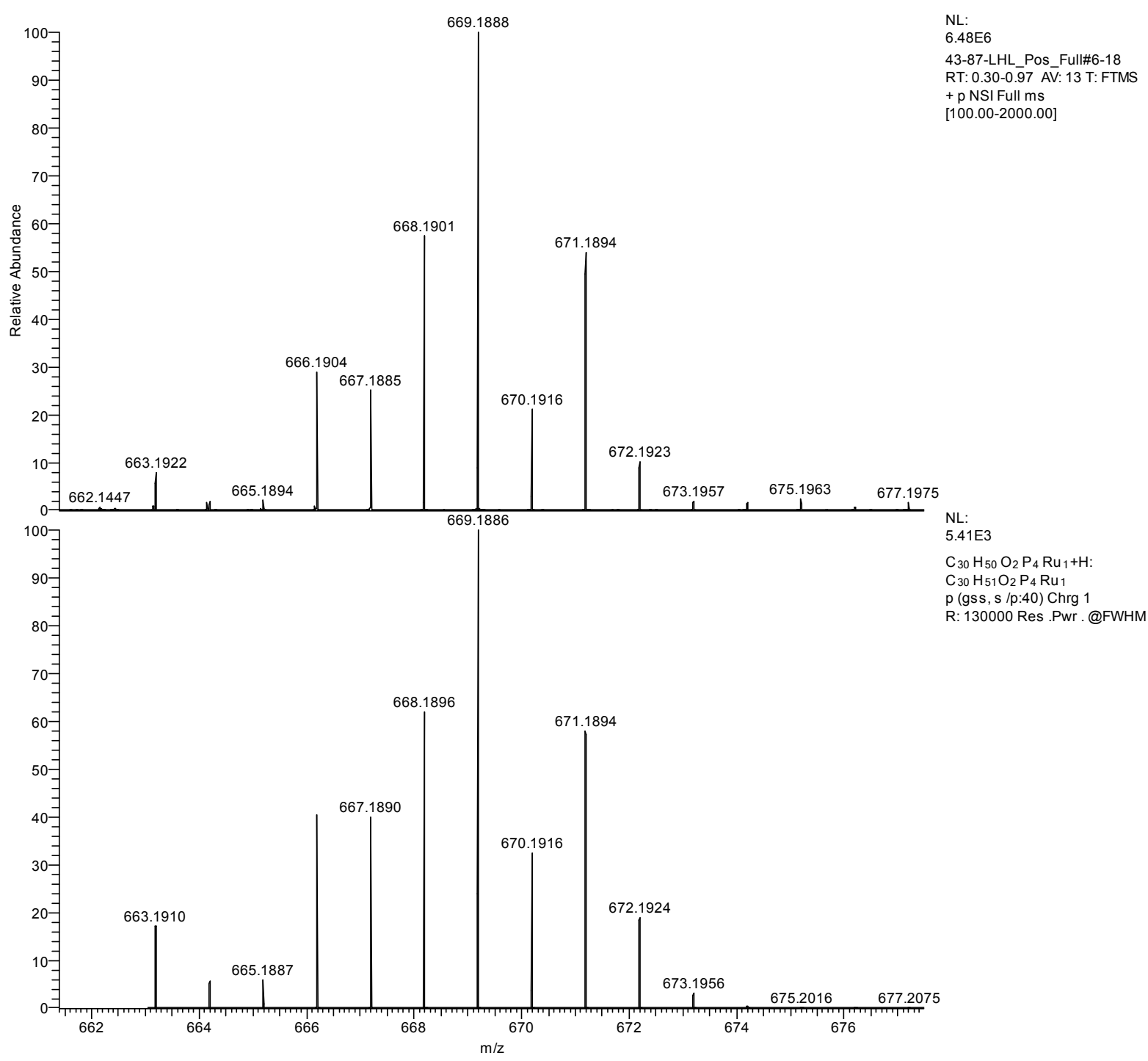


S2.9 cis/trans- $\left.\left[\mathrm{Ru}\left(\mathrm{C} \equiv \mathrm{CC}_{6} \mathrm{H}_{4}-4-\mathrm{CF}_{3}\right)_{2}\left(\mathrm{PMe}_{3}\right)_{4}\right]\right]_{3 \mathrm{C}}$

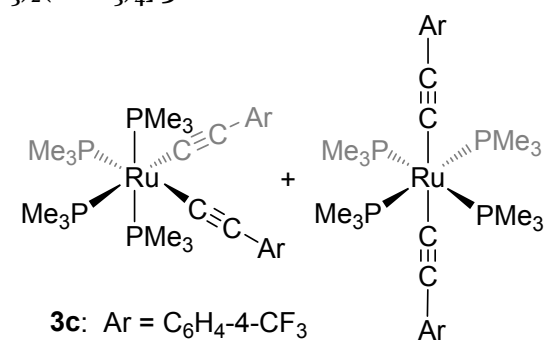

Figure S34. $_{31} \mathrm{P}\left\{{ }^{1} \mathrm{H}\right\}$ NMR spectrum for cis/trans- $\left[\mathrm{Ru}\left(\mathrm{C} \equiv \mathrm{CC}_{6} \mathrm{H}_{4}-4-\mathrm{CF}_{3}\right)_{2}\left(\mathrm{PMe}_{3}\right)_{4}\right]{ }_{3} \mathrm{C}$ (benzene- $\left.d_{6}, 162 \mathrm{MHz}\right)$ ${ }^{31} \mathrm{P}\left\{{ }^{1} \mathrm{H}\right\} \operatorname{NMR}\left(\mathrm{C}_{6} \mathrm{D}_{6}, 162 \mathrm{MHz}\right)$

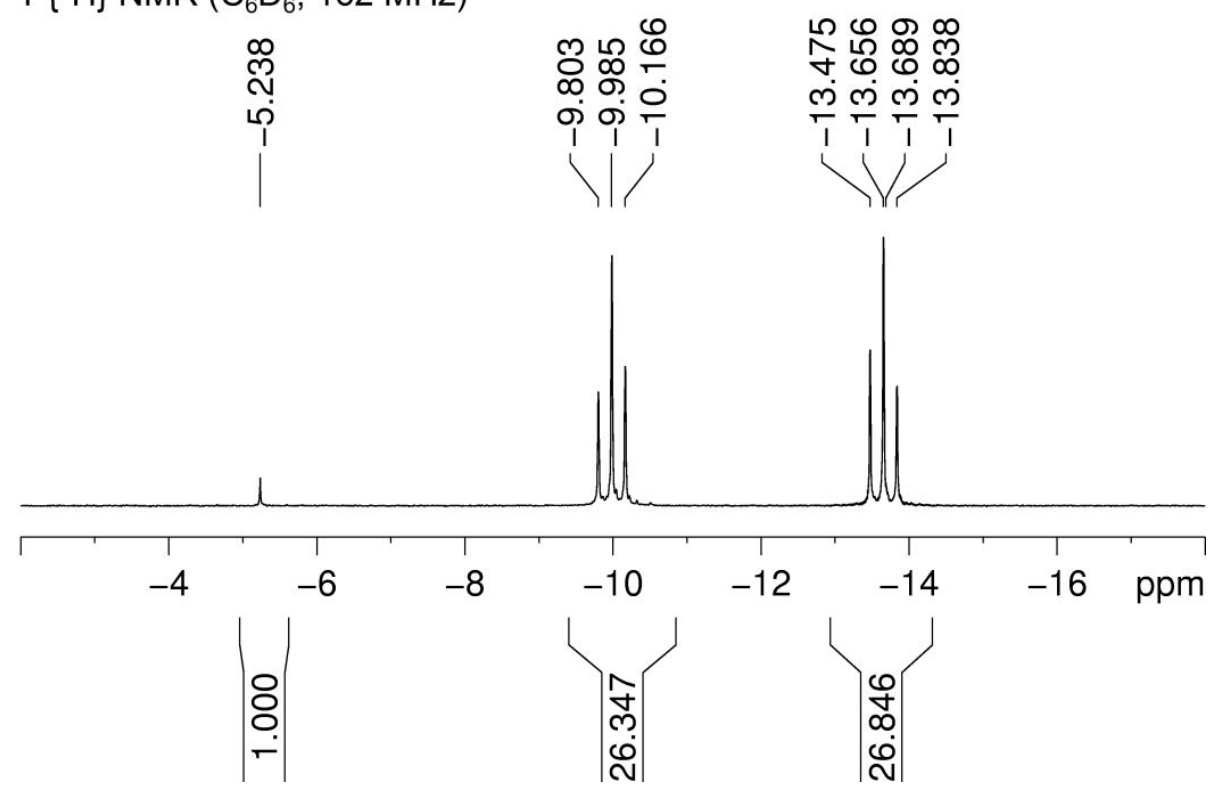

Figure $\mathrm{S}_{35} .{ }^{1} \mathrm{H}$ NMR spectrum for cis/trans- $\left[\mathrm{Ru}\left(\mathrm{C} \equiv \mathrm{CC}_{6} \mathrm{H}_{4}-4-\mathrm{CF}_{3}\right)_{2}\left(\mathrm{PMe}_{3}\right)_{4}\right]{ }_{3} \mathrm{C}$ (benzene- $\left.d_{6}, 400 \mathrm{MHz}\right)$

${ }^{1} \mathrm{H}$ NMR $\left(\mathrm{C}_{6} \mathrm{D}_{6}, 400 \mathrm{MHz}\right)$

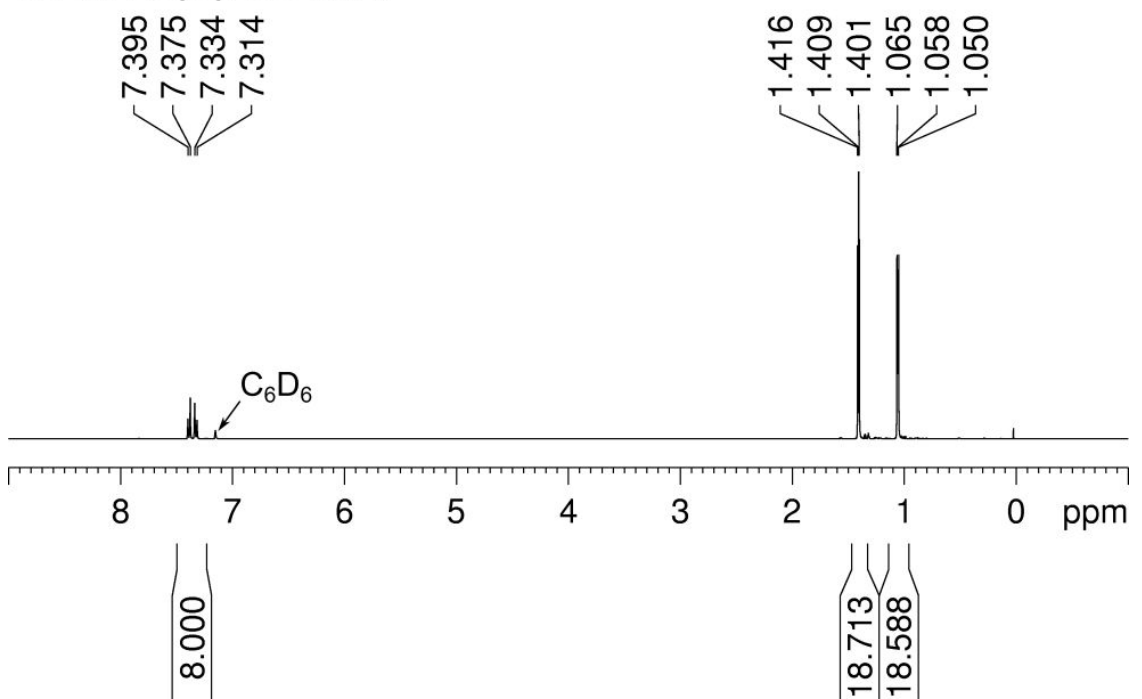


Figure $\mathrm{S}_{3} 6 .{ }^{13} \mathrm{C}\left\{{ }^{1} \mathrm{H}\right\}$ NMR spectrum for cis/trans- $\left[\mathrm{Ru}\left(\mathrm{C} \equiv \mathrm{CC}_{6} \mathrm{H}_{4}-4-\mathrm{CF}_{3}\right)_{2}\left(\mathrm{PMe}_{3}\right)_{4}\right] 3 \mathrm{c}$ (benzene- $\left.d_{6}, 101 \mathrm{MHz}\right)$ ${ }^{13} \mathrm{C}$ NMR $\left(\mathrm{C}_{6} \mathrm{D}_{6}, 101 \mathrm{MHz}\right)$

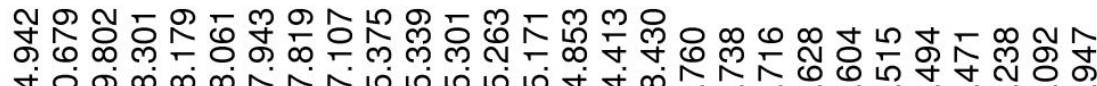

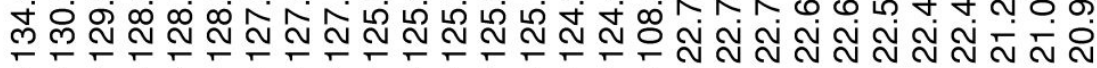

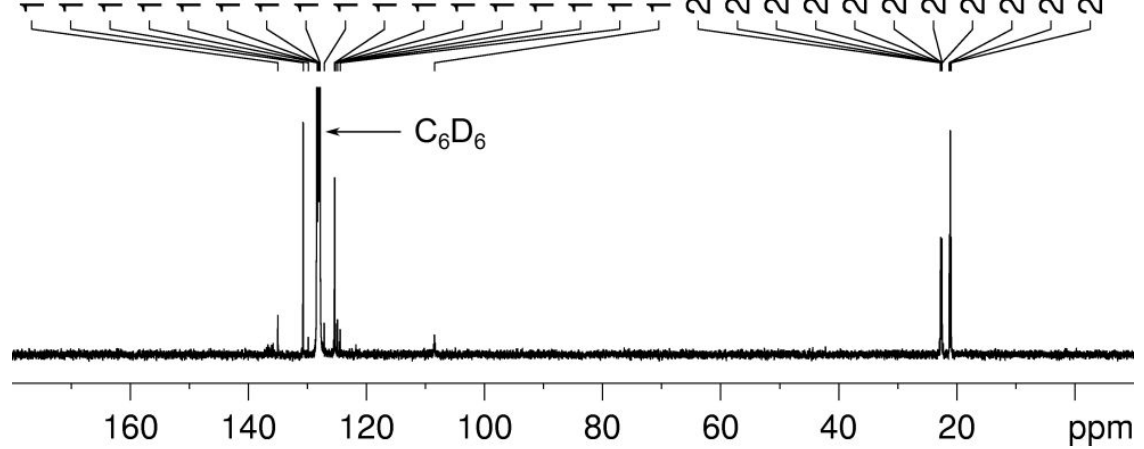

Figure S37. ${ }^{19} \mathrm{~F}$ NMR spectrum for cis/trans- $\left[\mathrm{Ru}\left(\mathrm{C} \equiv \mathrm{CC}_{6} \mathrm{H}_{4}-4-\mathrm{CF}_{3}\right)_{2}\left(\mathrm{PMe}_{3}\right)_{4}\right] 3 \mathrm{c}$ (benzene- $\left.d_{6}, 376 \mathrm{MHz}\right)$

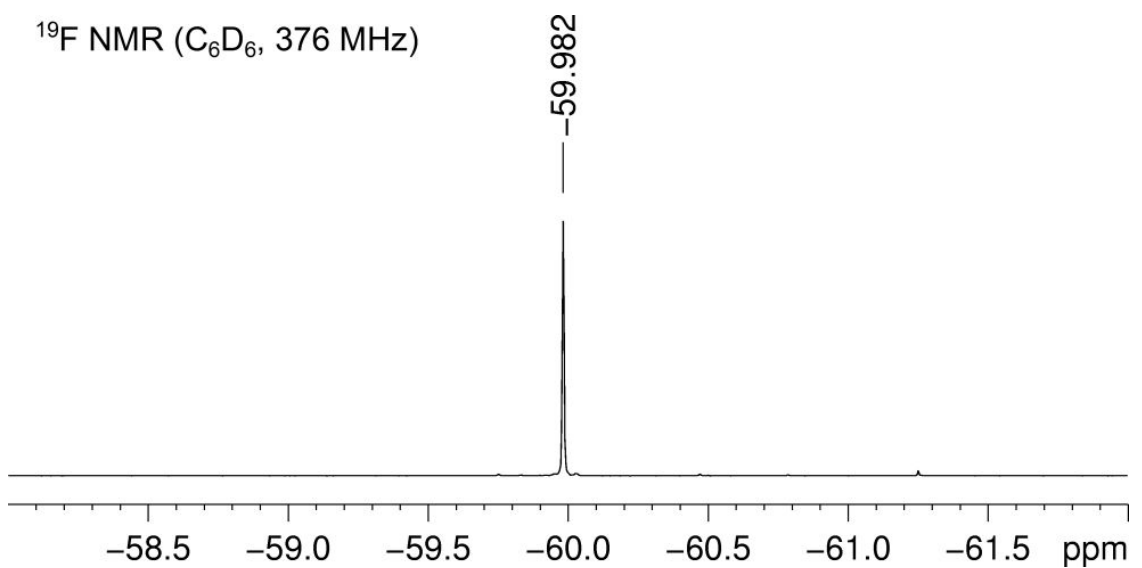


S2.10 Trans $-\left[\mathrm{Ru}\left(=\mathrm{C}=\mathrm{CH}\left(\mathrm{C}_{6} \mathrm{H}_{4}-4-\mathrm{OMe}\right)\right)\left(\mathrm{C} \mathrm{CC}_{6} \mathrm{H}_{4}-4-\mathrm{OMe}\right)\left(\mathrm{PMe}_{3}\right)_{4}\right]^{+} \mathrm{BF}_{4}^{-} 4^{-a}$.

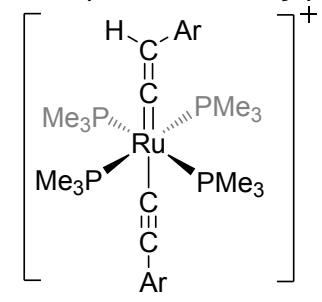

4a: $\mathrm{Ar}=\mathrm{C}_{6} \mathrm{H}_{4}-4-\mathrm{OMe}$

Figure $S_{3} 8 . \quad{ }^{31} \mathrm{P}\left\{{ }^{1} \mathrm{H}\right\} \quad \mathrm{NMR}$ spectrum for trans- $\left[\mathrm{Ru}\left(=\mathrm{C}=\mathrm{CH}\left(\mathrm{C}_{6} \mathrm{H}_{4}-4-\mathrm{OMe}\right)\right)\left(\mathrm{C} \equiv \mathrm{CC}_{6} \mathrm{H}_{4}-4-\mathrm{OMe}\right)\left(\mathrm{PMe}_{3}\right)_{4}\right]^{+} \mathrm{BF}_{4}^{-} \mathbf{4 a}^{\mathbf{a}}$ (acetone- $d_{6}, 162 \mathrm{MHz}$ )

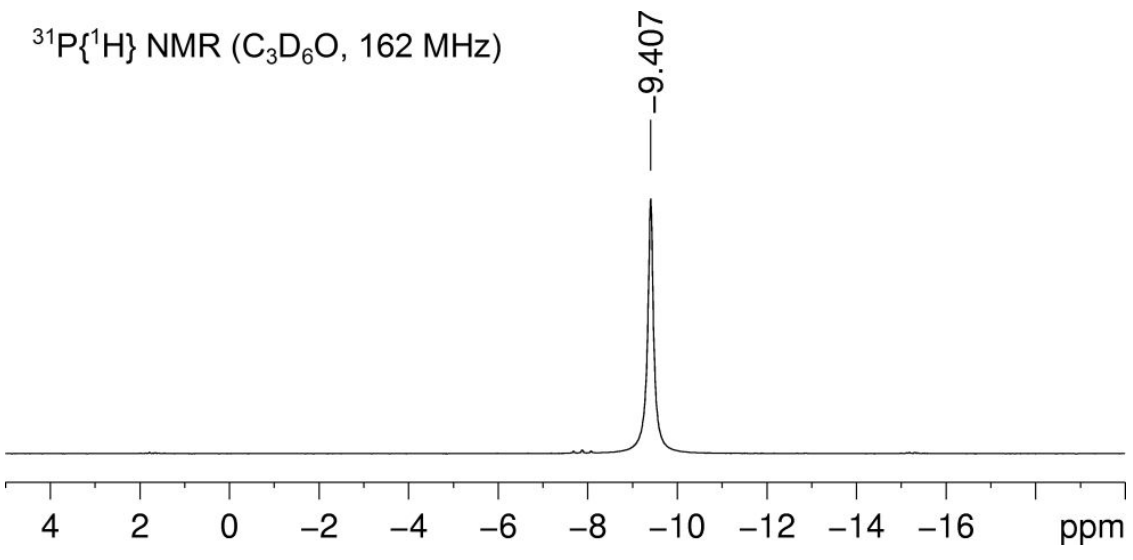

Figure S39. ${ }^{1} \mathrm{H}$ NMR spectrum for trans- $\left[\mathrm{Ru}\left(=\mathrm{C}=\mathrm{CH}\left(\mathrm{C}_{6} \mathrm{H}_{4}-4-\mathrm{OMe}\right)\right)\left(\mathrm{C} \equiv \mathrm{CC}_{6} \mathrm{H}_{4}-4-\mathrm{OMe}\right)\left(\mathrm{PMe}_{3}\right)_{4}\right]^{+} \quad \mathrm{BF}_{4}^{-} \quad \mathbf{4 a}^{\mathbf{a}}$ (acetone- $d_{6}, 400 \mathrm{MHz}$ )

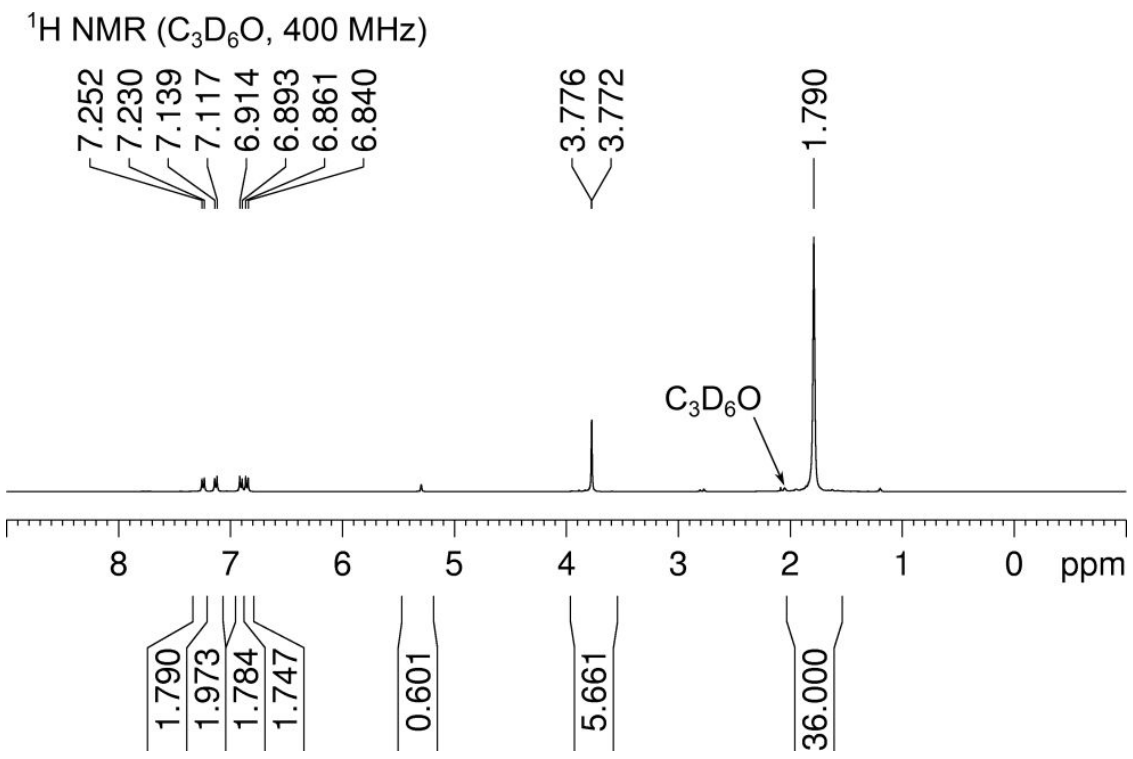


Figure S4o. ${ }^{13} \mathrm{C}\left\{{ }^{1} \mathrm{H}\right\} \quad \mathrm{NMR}$ spectrum for trans- $\left[\mathrm{Ru}\left(=\mathrm{C}=\mathrm{CH}\left(\mathrm{C}_{6} \mathrm{H}_{4}-4-\mathrm{OMe}\right)\right)\left(\mathrm{C} \equiv \mathrm{CC}_{6} \mathrm{H}_{4}-4-\mathrm{OMe}\right)\left(\mathrm{PMe}_{3}\right)_{4}\right]^{+} \mathrm{BF}_{4}^{-} \mathbf{4 a}^{\mathbf{a}}$ (acetone- $d_{6}, 101 \mathrm{MHz}$ )

${ }^{13} \mathrm{C}\left\{{ }^{1} \mathrm{H}\right\} \operatorname{NMR}\left(\mathrm{C}_{3} \mathrm{D}_{6} \mathrm{O}, 101 \mathrm{MHz}\right)$

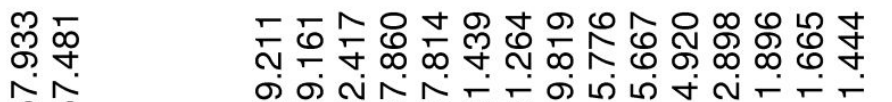
윰

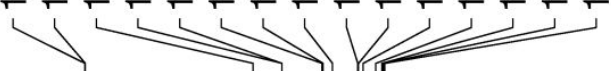

மூ น

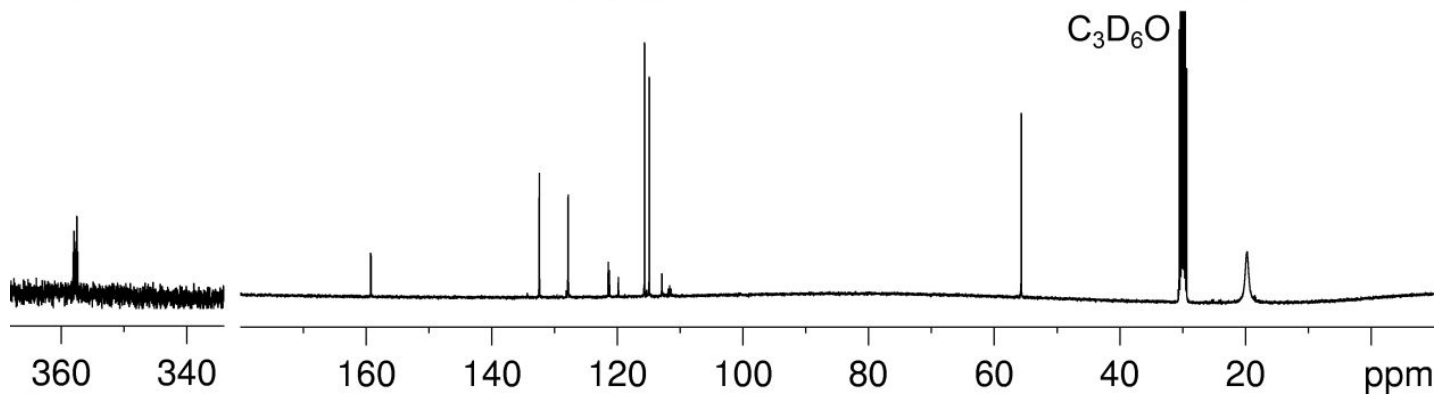

Figure S41. ${ }^{19} \mathrm{~F}$ NMR spectrum for trans- $\left[\mathrm{Ru}\left(=\mathrm{C}=\mathrm{CH}\left(\mathrm{C}_{6} \mathrm{H}_{4}-4-\mathrm{OMe}\right)\right)\left(\mathrm{C} \equiv \mathrm{CC}_{6} \mathrm{H}_{4}-4-\mathrm{OMe}\right)\left(\mathrm{PMe}_{3}\right)_{4}\right]^{+} \quad \mathrm{BF}_{4}^{-} \quad \mathbf{4 a}^{\mathbf{a}}$ (acetone- $d_{6}, 565 \mathrm{MHz}$ )

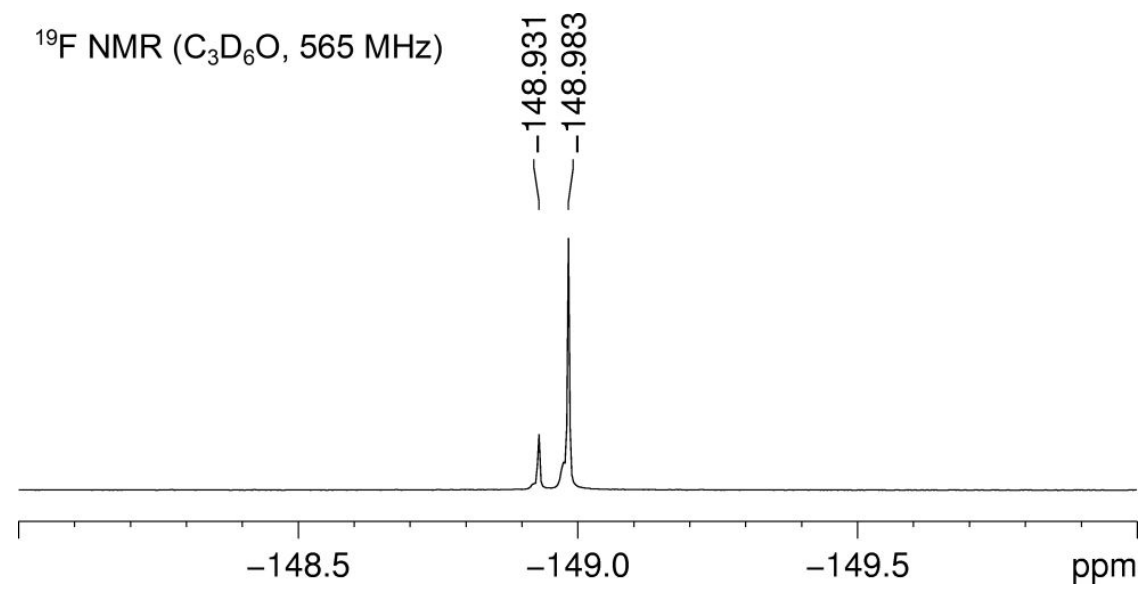


Figure $\mathrm{S}_{42}$. HRMS for trans- $\left[\mathrm{Ru}\left(=\mathrm{C}=\mathrm{CH}\left(\mathrm{C}_{6} \mathrm{H}_{4}-4-\mathrm{OMe}\right)\right)\left(\mathrm{C} \equiv \mathrm{CC}_{6} \mathrm{H}_{4}-4-\mathrm{OMe}\right)\left(\mathrm{PMe}_{3}\right)_{4}\right]+\mathrm{BF}_{4}^{-} 4 \mathbf{4}$ (acetonitrile, ESI) 45-101-LHL_Pos_Full \#21-32 RT: 0.60-0.93 AV: 12 NL: 4.76E8

T: FTMS + p NSI Full ms [200.00-2000.00]

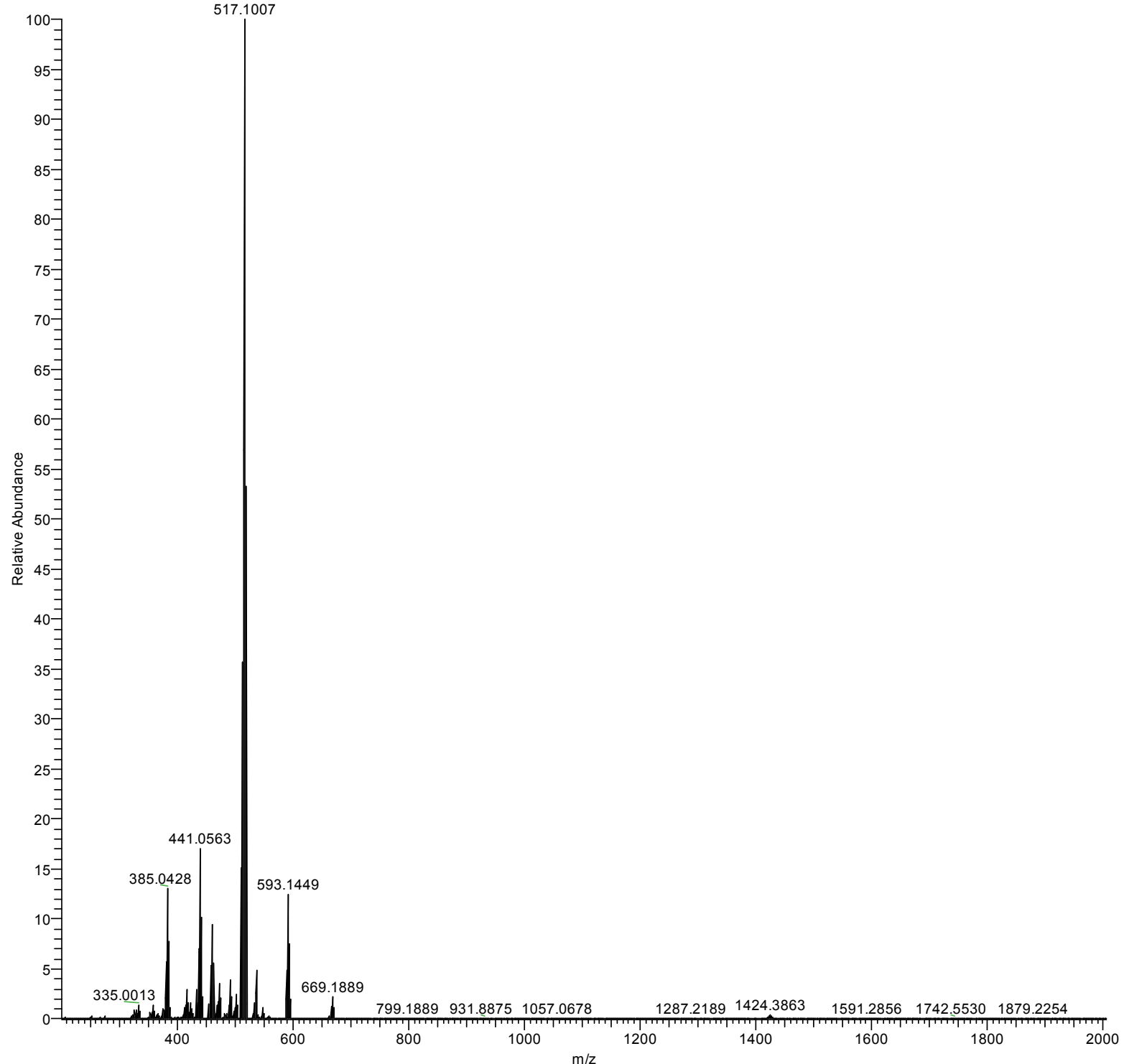


Figure S43. HRMS for trans- $\left[\mathrm{Ru}\left(=\mathrm{C}=\mathrm{CH}\left(\mathrm{C}_{6} \mathrm{H}_{4}-4-\mathrm{OMe}\right)\right)\left(\mathrm{C} \equiv \mathrm{CC}_{6} \mathrm{H}_{4}-4-\mathrm{OMe}\right)\left(\mathrm{PMe}_{3}\right)_{4}\right]^{+} \mathrm{BF}_{4}^{-} \quad$ 4a $^{\text {a }}$ (expansion, acetonitrile, ESI)

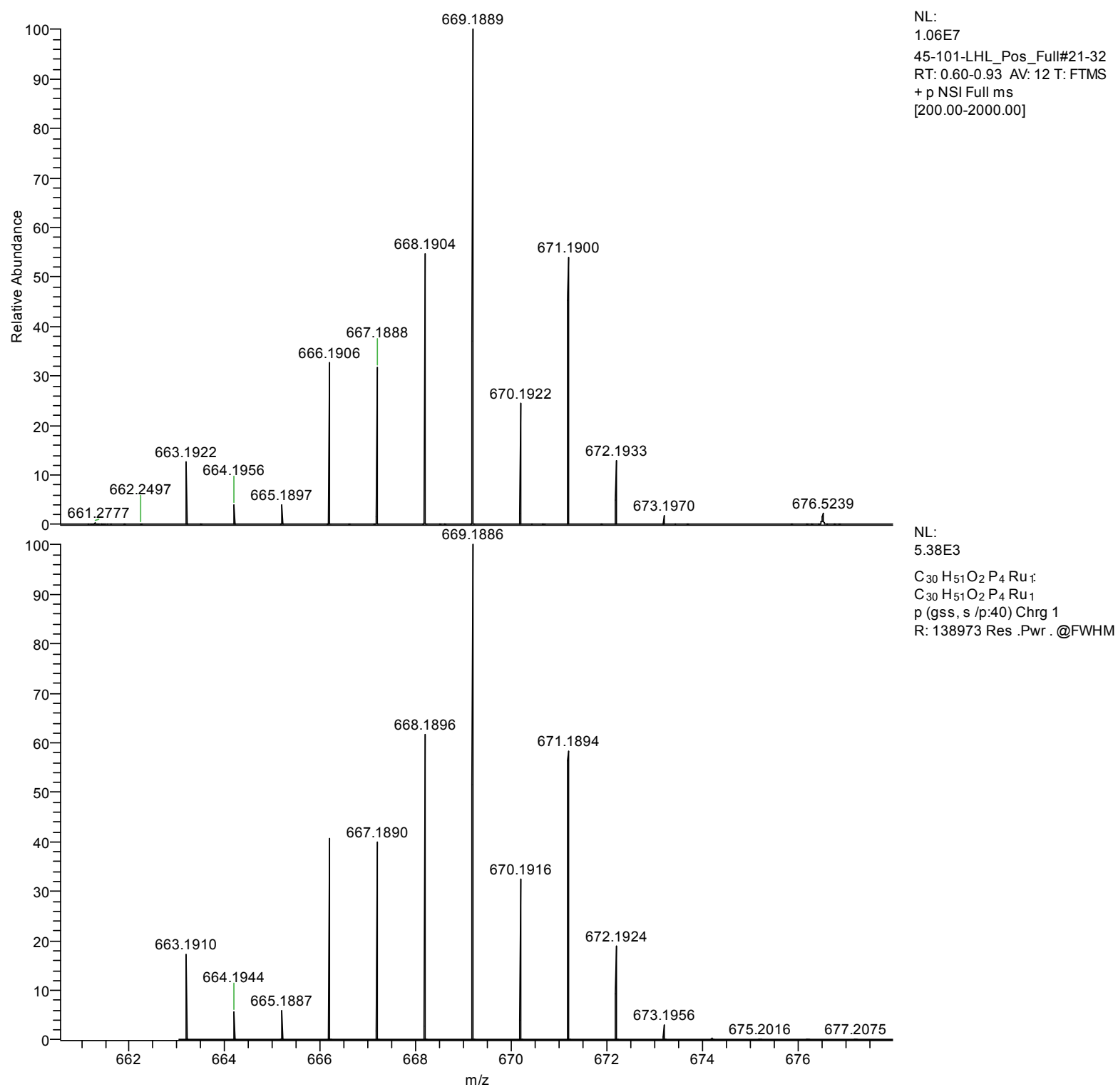




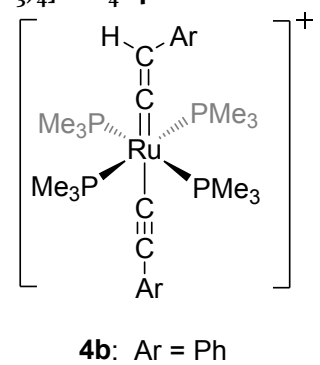

Figure S44. ${ }^{31} \mathrm{P}\left\{{ }^{1} \mathrm{H}\right\}$ NMR spectrum for trans- $\left[\mathrm{Ru}(=\mathrm{C}=\mathrm{CH}(\mathrm{Ph}))(\mathrm{C} \equiv \mathrm{CPh})\left(\mathrm{PMe}_{3}\right)_{4}\right]+\mathrm{BF}_{4}{ }_{4}^{-} \mathbf{b}$ (acetone- $\left.d_{6}, 243 \mathrm{MHz}\right)$

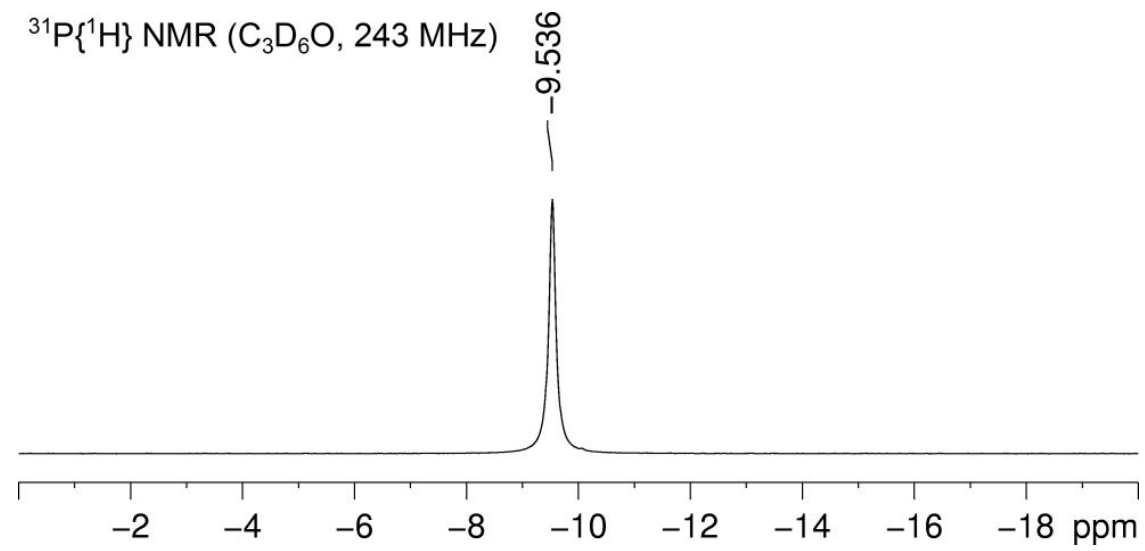

Figure S45. ${ }^{1} \mathrm{H}$ NMR spectrum for trans- $\left[\mathrm{Ru}(=\mathrm{C}=\mathrm{CH}(\mathrm{Ph}))(\mathrm{C} \equiv \mathrm{CPh})\left(\mathrm{PMe}_{3}\right)_{4}\right]+\mathrm{BF}_{4}{ }^{-} \mathbf{4} \mathbf{b}$ (acetone- $d_{6}$, 6oo $\left.\mathrm{MHz}\right)$ ${ }^{1} \mathrm{H}$ NMR $\left(\mathrm{C}_{3} \mathrm{D}_{6} \mathrm{O}, 600 \mathrm{MHz}\right)$

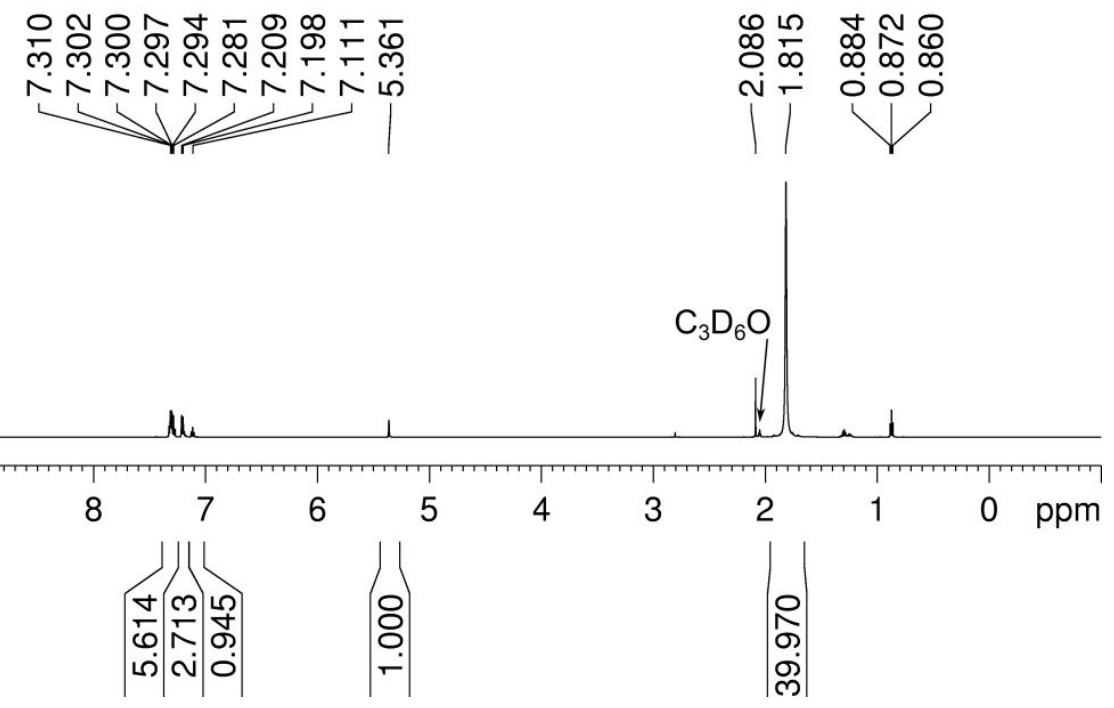


Figure S46. ${ }^{13} \mathrm{C}\left\{{ }_{1} \mathrm{H}\right\}$ NMR spectrum for trans $-\left[\mathrm{Ru}(=\mathrm{C}=\mathrm{CH}(\mathrm{Ph}))(\mathrm{C} \equiv \mathrm{CPh})\left(\mathrm{PMe}_{3}\right)_{4}\right]+\mathrm{BF}_{4}^{-} \mathbf{4}_{\mathbf{b}}$ (acetone- $\left.d_{6},{ }_{151} \mathrm{MHz}\right)$ ${ }^{13} \mathrm{C}\left\{{ }^{1} \mathrm{H}\right\} \operatorname{NMR}\left(\mathrm{C}_{3} \mathrm{D}_{6} \mathrm{O}, 151 \mathrm{MHz}\right)$

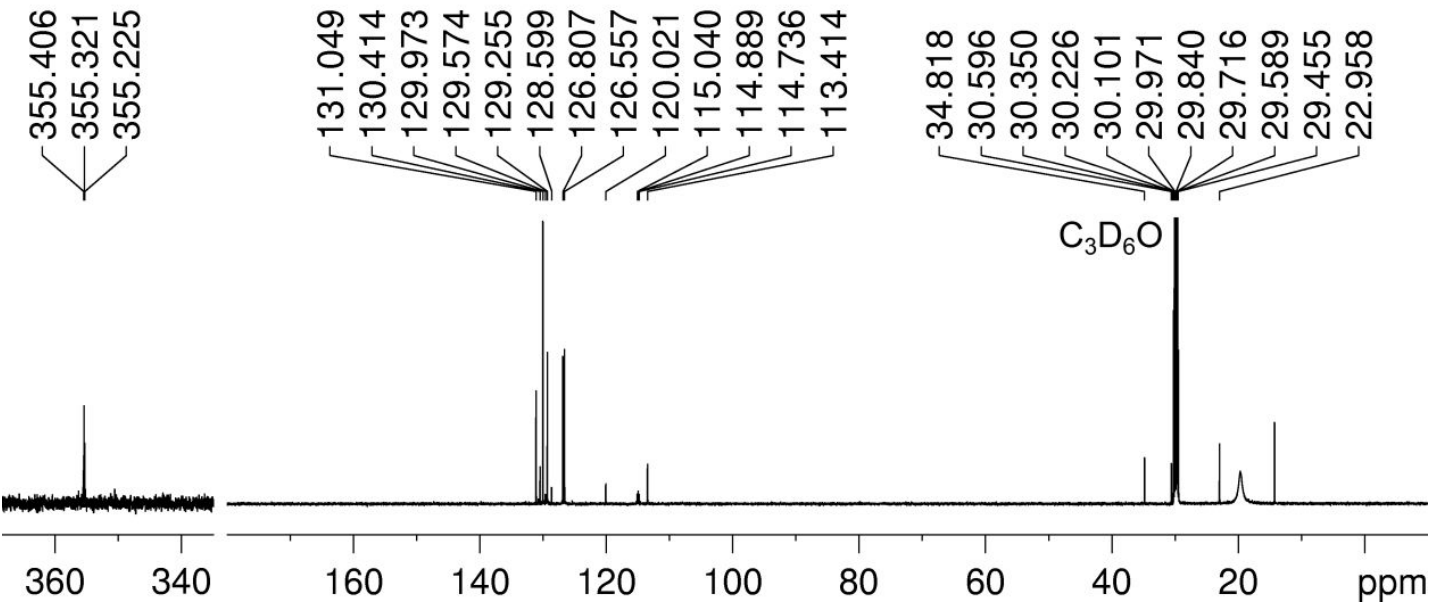

Figure S47. ${ }^{19} \mathrm{~F}$ NMR spectrum for trans- $\left[\mathrm{Ru}(=\mathrm{C}=\mathrm{CH}(\mathrm{Ph}))(\mathrm{C} \equiv \mathrm{CPh})\left(\mathrm{PMe}_{3}\right)_{4}\right]+\mathrm{BF}_{4}{ }_{4} \mathbf{4}^{\mathbf{b}}$ (acetone- $\left.d_{6},{ }_{565} \mathrm{MHz}\right)$

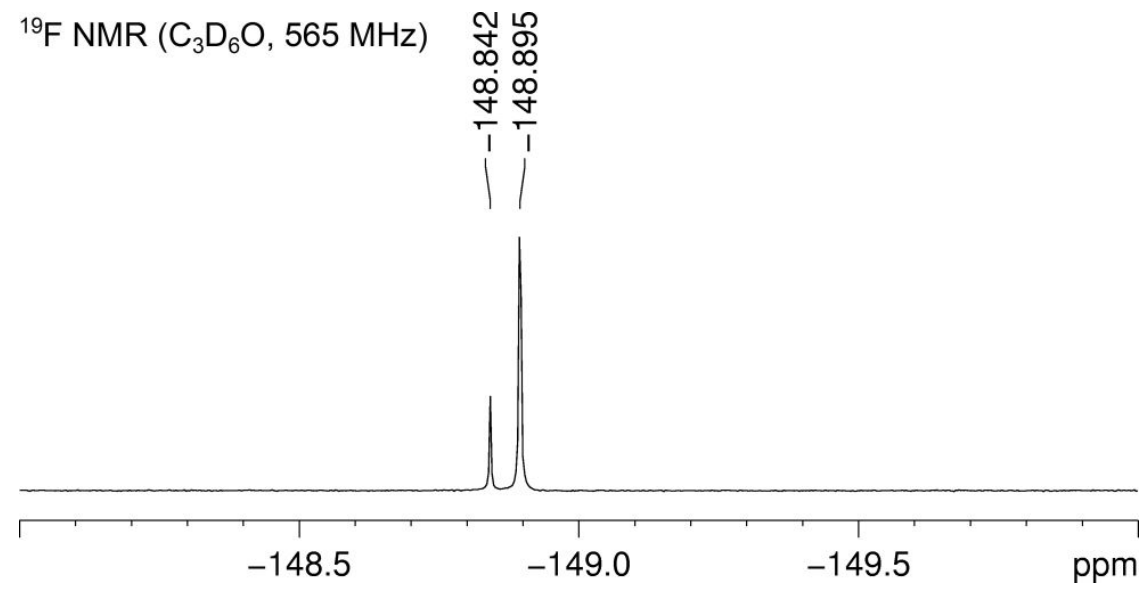


S3 $\mathrm{X}$-ray crystal structure of trans- $\left[\mathrm{Ru}\left(=\mathrm{C}=\mathrm{CH}\left(\mathrm{C}_{6} \mathrm{H}_{4}-4-\mathrm{OMe}\right)\right)\left(\mathrm{C} \equiv \mathrm{CC}_{6} \mathrm{H}_{4}-4-\mathrm{OMe}\right)\left(\mathrm{PMe}_{3}\right)_{4}\right]^{+} \mathrm{BF}_{4}{ }_{4}^{-}{ }_{4}^{\mathrm{a}}$

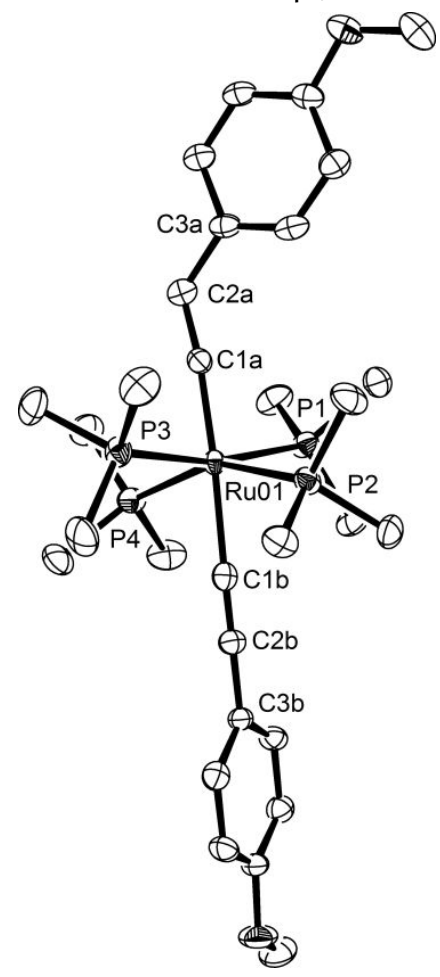

Figure S48. ORTEP plot of trans- $\left[\mathrm{Ru}\left(=\mathrm{C}=\mathrm{CH}\left(\mathrm{C}_{6} \mathrm{H}_{4}{ }^{-4}-\mathrm{OMe}\right)\right)\left(\mathrm{C} \equiv \mathrm{C}-\mathrm{C}_{6} \mathrm{H}_{4}-4-\mathrm{OMe}\right)\left(\mathrm{PMe}_{3}\right)_{4}\right]^{+} \mathrm{BF}_{4}^{-}{ }^{-}(\mathbf{4 a}),{ }_{50} \%$ ellipsoid probability, hydrogen atoms, acetone solvate and $\mathrm{BF}_{4}{ }_{4}$ counterion have been omitted for clarity. Selected bond lengths $(\AA)$ and angles $\left(^{\circ}\right)$ : Ruoi-P1 2.3744(7), Ruoi-P2 2.3984(7), Ruo1-P3 2.3699(7), Ruo1-P4 2.3647(7), Ruoi-Cra 1.881(2), Ruo1-C1b 2.086(2), C2a-C1a 1.325(3), C1b-C2b 1.214(3), C2a-C3a 1.476(4), C2b-C3b 1.443(3), C2a-C1a-Ruo1 172.5(2), C2b-C1b-Ruo1 174.1(2), C1a-C2a-C3a 126.9(3), C1b-C2b-C3b 175.3(3). 
Table S1. Crystallographic data for $\left[\mathrm{Ru}\left(\eta^{3}-\mathrm{CF}_{3}-4-\mathrm{C}_{6} \mathrm{H}_{4} \mathrm{C} \equiv \mathrm{C}-\mathrm{C}=\mathrm{CH}\left(\mathrm{C}_{6} \mathrm{H}_{4}-4-\mathrm{CF}_{3}\right)\right)\left(\mathrm{PMe}_{3}\right)_{4}\right]^{+} \mathrm{BF}_{4}^{-}(\mathbf{1 c}),\left[\mathrm{Ru}\left(\mathrm{MeO}-4-\mathrm{C}_{6} \mathrm{H}_{4} \mathrm{C} \equiv \mathrm{C}-\right.\right.$ $\left.\mathrm{C}=\mathrm{CH}\left(\mathrm{C}_{6} \mathrm{H}_{3}-4-\mathrm{OMe}\right)\left(\mathrm{PMe}_{3}\right)_{4}\right]$ (2a), cis- $\left[\mathrm{Ru}\left(\mathrm{C} \equiv \mathrm{CC}_{6} \mathrm{H}_{4}-4-\mathrm{CF}_{3}\right)_{2}\left(\mathrm{PMe}_{3}\right)_{4}\right]$ (3c), trans- $\left[\mathrm{Ru}\left(=\mathrm{C}=\mathrm{CH}\left(\mathrm{C}_{6} \mathrm{H}_{4}-4-\mathrm{OMe}\right)\right)\left(\mathrm{C} \equiv \mathrm{C}-\mathrm{C}_{6} \mathrm{H}_{4}-4-\right.\right.$ $\left.\mathrm{OMe})\left(\mathrm{PMe}_{3}\right)_{4}\right]^{+} \mathrm{BF}_{4}^{-}(\mathbf{4 a})$ and trans $-\left[\mathrm{Ru}(=\mathrm{C}=\mathrm{CH}(\mathrm{Ph}))(\mathrm{C} \equiv \mathrm{CPh})\left(\mathrm{PMe}_{3}\right)_{4}\right]^{+} \mathrm{BF}_{4}^{-}(\mathbf{4} \mathbf{b})$.

\begin{tabular}{|c|c|c|c|c|c|}
\hline & 1C & $2 a$ & $3 c$ & $4 a$ & $4 b$ \\
\hline CCDC Number & 1966218 & 1966219 & 1966220 & 1966222 & 1966221 \\
\hline Formula & $\mathrm{C}_{30} \mathrm{H}_{45} \mathrm{BF}_{10} \mathrm{P}_{4} \mathrm{Ru}$ & $\mathrm{C}_{30} \mathrm{H}_{50} \mathrm{O}_{2} \mathrm{P}_{4} \mathrm{Ru}$ & $\mathrm{C}_{30} \mathrm{H}_{44} \mathrm{~F}_{6} \mathrm{P}_{4} \mathrm{Ru}$ & $\mathrm{C}_{33} \mathrm{H}_{57} \mathrm{BF}_{4} \mathrm{O}_{3} \mathrm{P}_{4} \mathrm{Ru}$ & $\mathrm{C}_{28.75} \mathrm{H}_{48.8} \mathrm{BF}_{4} \mathrm{P}_{4} \mathrm{Ru}$ \\
\hline $\mathrm{M}\left(\mathrm{g} \mathrm{mol}^{-1}\right)$ & 831.42 & 667.65 & 743.60 & 813.54 & 706.23 \\
\hline Size $\left(\mathrm{mm}^{3}\right)$ & $\begin{array}{c}0.157 \times 0.115 \times \\
0.067\end{array}$ & $\begin{array}{c}0.340 \times 0.110 \times \\
0.030\end{array}$ & $\begin{array}{c}0.217 \times 0.074 \times \\
0.052\end{array}$ & $\begin{array}{c}0.235 \times 0.219 \times \\
0.074\end{array}$ & $0.24 \times 0.12 \times 0.06$ \\
\hline $\begin{array}{c}\text { Crystal } \\
\text { morphology }\end{array}$ & Yellow plate & Yellow plate & Colourless plate & Purple plate & Pink plate \\
\hline Crystal system & orthorhombic & monoclinic & monoclinic & monoclinic & monoclinic \\
\hline Space group & P21 2121 & $\mathrm{P} 21 / \mathrm{c}$ & P1 21/c 1 & $\mathrm{P}_{1} 21 / \mathrm{n}_{1}$ & $\mathrm{P}_{1} 21 / \mathrm{C}_{1}$ \\
\hline $\mathrm{a}(\AA)$ & $10.4300(8)$ & $14.2227(5)$ & $14.201(2)$ & $13.1603(11)$ & $12.6906(9)$ \\
\hline $\mathrm{b}(\AA)$ & $14.7049(11)$ & $9.1520(3)$ & $15 \cdot 580(3)$ & $22.3645(17)$ & $14.1339(10)$ \\
\hline c $(\AA)$ & $24 \cdot 383(2)$ & $24.9166(8)$ & $17.065(3)$ & $14.1432(11)$ & $22.3102(16)$ \\
\hline$\alpha\left(^{\circ}\right)$ & 90 & 90 & 90 & 90 & 90 \\
\hline$\beta\left(^{\circ}\right)$ & 90 & $98.755(2)$ & $109 \cdot 975(7)$ & $107 \cdot 334(2)$ & $98.455(4)$ \\
\hline$\gamma\left({ }^{\circ}\right)$ & 90 & 90 & 90 & 90 & 90 \\
\hline $\mathrm{V}\left(\AA^{\circ} 3\right)$ & $3739 \cdot 7(5)$ & $3205 \cdot 51(19)$ & $3548.5(10)$ & $3973.6(5)$ & $3958.2(5)$ \\
\hline $\mathrm{Z}$ & 4 & 4 & 4 & 4 & 4 \\
\hline $\mathrm{D}_{\mathrm{c}}\left(\mathrm{g} / \mathrm{cm}^{3}\right)$ & 1.477 & 1.383 & 1.392 & 1.360 & 1.185 \\
\hline$\mu\left(\mathrm{mm}^{-1}\right)$ & 0.660 & 0.714 & 0.672 & 0.605 & 0.592 \\
\hline $\mathrm{F}(\mathrm{ooo})$ & 1696 & 1400 & 1528 & 1696 & 1465 \\
\hline$\theta_{\max }\left({ }^{\circ}\right)$ & 24.999 & 26.20 & 27.036 & 27.445 & 24.999 \\
\hline $\mathrm{N}$ & 35929 & 211141 & 27568 & 110887 & 65572 \\
\hline $\mathrm{N}_{\text {ind }}$ & $\begin{array}{c}6580 \\
\left(\mathrm{R}_{\text {int }}=0.1387\right)\end{array}$ & $\begin{array}{c}6625 \\
\left(R_{\text {int }}=0.1448\right)\end{array}$ & $\begin{array}{c}7641 \\
\left(R_{\text {int }}=0.1000\right)\end{array}$ & $\begin{array}{c}9127 \\
\left(R_{\text {int }}=0.0561\right)\end{array}$ & $\begin{array}{c}6968 \\
\left(R_{\text {int }}=0.0788\right)\end{array}$ \\
\hline Goodness of fit & 1.139 & 1.034 & 1.009 & 1.044 & 1.115 \\
\hline $\begin{array}{l}\text { Final } R \text { indexes } \\
\qquad(\mathrm{I}>\mathbf{2} \sigma(\mathrm{I}))\end{array}$ & $\begin{array}{c}R_{1}=0.0890 \\
w R_{2}=0.2180\end{array}$ & $\begin{array}{c}\mathrm{R}_{1}=0.0308 w \mathrm{R}_{2}= \\
0.0592\end{array}$ & $\begin{array}{c}R_{1}=0.0690 \\
w R_{2}=0.1649\end{array}$ & $\begin{array}{c}\mathrm{R}_{1}=0.0356 \\
\mathrm{wR}_{2}=0.0804\end{array}$ & $\begin{array}{c}R_{1}=0.0587 \\
w R_{2}=0.1634\end{array}$ \\
\hline $\begin{array}{l}\text { Final } \mathrm{R} \text { indexes } \\
\text { (all data) }\end{array}$ & $\begin{array}{c}R_{1}=0.0997 \\
w R_{2}=0.2257\end{array}$ & $\begin{aligned} \mathrm{R}_{1}= & 0.0517 \mathrm{wR}_{2} \\
= & 0.0671\end{aligned}$ & $\begin{array}{c}R_{1}=0.1286 \\
w R_{2}=0.1945\end{array}$ & $\begin{array}{c}R_{1}=0.0464 \\
w R_{2}=0.0879\end{array}$ & $\begin{array}{c}\mathrm{R}_{1}=0.0771 \\
\mathrm{wR}_{2}=0.1788\end{array}$ \\
\hline
\end{tabular}

For structure $3 \mathrm{c}$, one of the $\mathrm{PMe}_{3}$ groups and both the $\mathrm{CF}_{3}$ groups exhibited orientational disorders, which were modelled over two and three (for one of the $\mathrm{CF}_{3}$ groups) positions with appropriate occupancies. Restrainsts DELU / RIGU/ EADP (as in Shelxl software) ${ }^{1}$ were applied wherever necessary to keep the anisotropic displacments of these partially occupied moieties within reasonable limits.

For structure $\mathbf{4} \mathbf{b}$, the terminal phenyl group atoms of the linear $\mathrm{C}-\mathrm{C}$-Ph group showed higher anisotropy; the atoms of this ring were then split over two equally occupied sites. The $\mathrm{BF}_{4}$ anion was also modelled for disorder with two equally occupied orientations. The geometry of the different parts was restrained to be similar by using command SADI and the anisotropic displacments were restrained by the same commands RIGU and EADP as in 3c, as per the Shelxl least-squares refinement software. ${ }^{1}$

(1) Sheldrick, G. Crystal structure refinement with SHELXL. Acta Crystallogr., Sect. C: Cryst. Struct. Commun. 2015, $71,3-8$. 